\title{
Recent developments in the evaluation of the 3D fundamental solution and its derivatives for transversely isotropic elastic materials*
}

\author{
V. Mantič, L. Távara, J.E. Ortiz, F. París \\ Group of Elasticity and Strength of Materials, School of Engineering \\ University of Seville, Camino de los Descubrimientos s/n, 41092 Sevilla, Spain \\ mantic@esi.us.es, ltavara@esi.us.es, jortiz@esi.us.es, paris@esi.us.es
}

\begin{abstract}
.
Explicit closed-form real-variable expressions of a fundamental solution and its derivatives for three-dimensional problems in transversely linear elastic isotropic solids are presented. The expressions of the fundamental solution in displacements $U_{i k}$ and its derivatives, originated by a unit point force, are valid for any combination of material properties and for any orientation of the radius vector between the source and field points. An expression of $U_{i k}$ in terms of the Stroh eigenvalues on the oblique plane normal to the radius vector is used as starting point. Working from this expression of $U_{i k}$, a new approach (based on the application of the rotational symmetry of the material) for deducing the first and second order derivative kernels, $U_{i k, j}$ and $U_{i k, j \ell}$ respectively, has been developed. The expressions of the fundamental solution and its derivatives do not suffer from the difficulties of some previous expressions, obtained by other authors in different ways, with complex valued functions appearing for some combinations of material parameters and/or with division by zero for the radius vector at the rotational symmetry axis. The expressions of $U_{i k}, U_{i k, j}$ and $U_{i k, j \ell}$ are presented in a form suitable for an efficient computational implementation in BEM codes.
\end{abstract}

Keywords: transversely isotropic materials, Stroh formalism, fundamental solution, free-space Green's functions, Somigliana identity, boundary integral equations, boundary element method

\section{Introduction}

A comprehensive review of the history of the development of fundamental solutions and their derivatives for general anisotropic materials, and in particular for the transversely isotropic materials, was recently presented in $[1,2]$. Thus, only the most relevant contributions closely related to the present work will be briefly mentioned.

\footnotetext{
*Extended version of the Keynote lecture by V. Mantič presented at the International Conference on Boundary Element Techniques IX (BETEQ) in Seville on 10 July, 2008.
} 
Regarding anisotropic elastic materials, the expression of $U_{i k}(i, k=1,2,3)$ obtained by Fredholm [3] using a 3D Fourier transform was used in the past as the basis for different contributions aimed to obtain $U_{i k}$ expressions as simple and explicit as possible, e.g. Lifshitz and Rozentsweig [4].

The application of the Stroh formalism to anisotropic elasticity, see Ting [5], to evaluate $U_{i k}$ and its derivatives in 3D has shown to be an important approach that leaded to several substantial contributions starting in the 1970s with Malén [6] and Barnett [7], and more recently, Nakamura and Tanuma [8], Ting and Lee [9], Lee [10, 11] and Tanuma [12]. An implementation in 3-D BEM of expressions of anisotropic fundamental solutions and its derivatives using the Stroh formalism, as introduced by Lee [10], was recently developed by Shiah et. al. [13, 15], Tan et. al. [14] and Buroni et. al. [16].

In the specific case of transversely isotropic materials the expressions deduced by Pan and Chou [17] are usually used in BEM codes [18, 19]. Loloi [20] and Ariza and Domínguez [23] presented the expressions of the strongly singular and hypersingular kernel, respectively, obtained from Pan and Chou's [17] solution. This solution [17] has several features which make somewhat cumbersome its implementation covering all possible cases: $i$ ) expressions depending on the values of $\triangle=\sqrt{C_{11} C_{33}}-C_{13}-2 C_{44}$ (positive, negative or zero) and in particular its complex-variable character for $\triangle<0$; ii) a loss of precision and/or a division by zero for the spherical angle $\phi=\pi$ or $\phi=0$. In the previous expressions it is assumed that the $x_{3}$-axis is the rotational symmetry axis of the transversal isotropic material. Although the difficulty with the degeneracy problem at $\phi=\pi$ has been solved by Loloi [20] by means of an ad hoc approach, the mentioned features may still cause some difficulties in using this expression in further analytic deductions and in BEM development. Other authors as, Ding et. al. [21] also presented a fundamental solution for transversely isotropic media with complex-variable character. While, Yue [22] presented the fundamental solutions in displacement and tractions for different bimaterial systems, that can be used in the displacement boundary integral equation.

The aim of the present work is to obtain completely general and closed-form realvariable expressions of $U_{i k}(\boldsymbol{x})$ and its first and second order derivatives, $U_{i k, j}(\boldsymbol{x})$ and $U_{i k, j l}(\boldsymbol{x})(i, j, k, l=1,2,3)$, valid for any transversely isotropic material and simpler than the available expressions of the fundamental solution and its derivatives, with an arbitrary spatial orientation and allowing for an easy and efficient computational implementation. The use of $U_{i k}(x)$ expression introduced by Ting and Lee [9] and the transformation rule introduced therein, that was corrected and extended in [1], are crucial for the achievement of this aim.

\section{Fundamental solution and its derivatives for linear elastic anisotropic materials}

Consider a cartesian coordinate system $\left(x_{1}, x_{2}, x_{3}\right)$ with the origin $O$ and the corresponding spherical coordinate system $(r, \theta, \phi)$, see Fig. 1 .

The fundamental solution in displacements $U_{i k}$ originated by the unit concentrated force at $O$, and its derivatives, can be expressed in terms of the so-called modulation 
functions depending only on the spherical angles $\theta$ and $\phi$ in the following form:

$$
\begin{aligned}
U_{i k}(r, \theta, \phi) & =\frac{1}{4 \pi r} \widehat{U}_{i k}(\theta, \phi), \\
U_{i k, j}(r, \theta, \phi) & =\frac{1}{4 \pi r^{2}} \widehat{U}_{i k ; j}(\theta, \phi), \\
U_{i k, j \ell}(r, \theta, \phi) & =\frac{1}{4 \pi r^{3}} \widehat{U}_{i k ; j \ell}(\theta, \phi),
\end{aligned}
$$

where $i, j, k, l=1,2,3$ and 'widehat' symbol denotes the modulation functions of the fundamental solution, and also of its derivatives, with respect to cartesian coordinates. The modulation functions are independent of the radius $r$ in spherical coordinates. While a comma between subscripts denotes a derivative with respect to a cartesian coordinate, a semicolon in a modulation function is used only (mnemotechnically) to represent that this function is associated to the corresponding derivative kernel in (2) or (3).

Malén [6] and others showed that

$$
\widehat{U}_{i k}(\theta, \phi)=H_{i k}(\theta, \phi),
$$

where $H_{i k}$ is the Barnett-Lothe tensor of the Stroh formalism of the anisotropic elasticity. A novel approach for obtaining an analytic expression of $H_{i k}$ was introduced by Ting and Lee [9]. By applying standard rules for differentiation with respect to cartesian coordinates in (2) and (3) we obtain

$$
\begin{aligned}
\widehat{U}_{i k ; j}(\theta, \phi)= & H_{i k, j}(r, \theta, \phi) r-H_{i k}(\phi, \theta) r,{ }_{j}, \\
\widehat{U}_{i k ; j \ell}(\theta, \phi)= & H_{i k, j \ell}(r, \theta, \phi) r^{2}-H_{i k, j}(r, \theta, \phi) r, \ell-H_{i k, \ell}(r, \theta, \phi) r_{, j} r \\
& -H_{i k}(\theta, \phi)\left(\delta_{j \ell}-3 r_{, j} r_{, \ell}\right),
\end{aligned}
$$

where $r_{, j}(\theta, \phi)=r_{j}(r, \theta, \phi) / r$.

By conveniently introducing new modulation functions obtained from the derivatives of $H_{i k}(\theta, \phi)$ as

$$
\begin{aligned}
H_{i k, j}(r, \theta, \phi) & =r^{-1} \widehat{H}_{i k ; j}(\theta, \phi), \\
H_{i k, j \ell}(r, \theta, \phi) & =r^{-2} \widehat{H}_{i k ; j \ell}(\theta, \phi),
\end{aligned}
$$

the modulation functions of the derivatives of $U_{i k}$ can be expressed in terms of the modulation functions $H_{i k}, \widehat{H}_{i k ; j}$ and $\widehat{H}_{i k ; j \ell}$,

$$
\begin{aligned}
\widehat{U}_{i k ; j}(\theta, \phi)= & \widehat{H}_{i k ; j}(\theta, \phi)-H_{i k}(\theta, \phi) r_{, j}, \\
\widehat{U}_{i k ; j \ell}(\theta, \phi)= & \widehat{H}_{i k ; j \ell}(\theta, \phi)-\widehat{H}_{i k ; j}(\theta, \phi) r_{, \ell}-\widehat{H}_{i k ; \ell}(\theta, \phi) r_{, j} \\
& -H_{i k}(\theta, \phi)\left(\delta_{j \ell}-3 r_{, j} r_{, \ell}\right) .
\end{aligned}
$$

Thus, in order to achieve expressions of the modulations functions $\widehat{U}_{i k}, \widehat{U}_{i k ; j}$ and $\widehat{U}_{i k ; j \ell}$ suitable for implementation in BEM codes we need to deduce general and as simple and compact as possible expressions of modular functions $H_{i k}, \widehat{H}_{i k ; j}$ and $\widehat{H}_{i k ; j \ell}$. As will be shown, for transversely isotropic materials simple and compact expressions of these modulation functions are only needed for a particular coordinate plane including the $x_{3}$ axis, the plane $x_{2}=0$ being used in the present work. 


\section{Modulation function of the fundamental solution and of its deriva- tives for linear elastic transversally isotropic materials}

\subsection{Transformation laws}

The key idea in the present approach for the evaluation of the modulation functions $\widehat{U}_{i k ; j}$ and $\widehat{U}_{i k ; j \ell}$ is to generalize the procedure proposed by Ting and Lee [9] for the evaluation of $\widehat{U}_{i k}=H_{i k}$. This procedure will be sketched briefly in what follows. Let us define a vector $\widehat{\boldsymbol{x}}$ corresponding to a vector $\boldsymbol{x}=\left(x_{1}, x_{2}, x_{3}\right)$ as

$$
\widehat{\boldsymbol{x}}=\left(r_{12}, 0, x_{3}\right), \quad \text { where } \quad r_{12}=\sqrt{x_{1}^{2}+x_{2}^{2}} .
$$

Let $c=\cos \phi=x_{3} / r$ and $s=\sin \phi=r_{12} / r$, the angle $\phi, 0 \leq \phi \leq \pi$, being shown in Fig. 1. Then, defining $\boldsymbol{n}=(c, 0,-s)$ and $\boldsymbol{m}=(0,1,0),[\boldsymbol{n}, \boldsymbol{m}, \widehat{\boldsymbol{x}} / r]$ forms a righthanded triad.

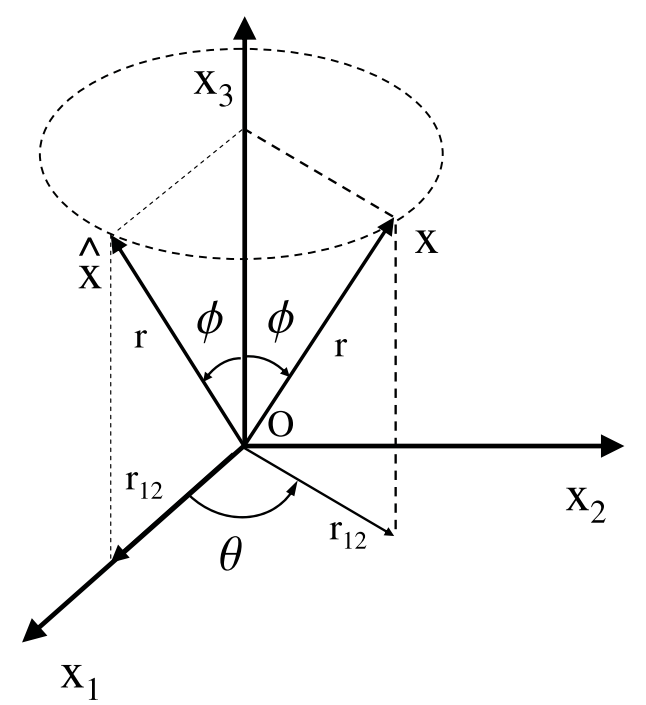

Figure 1: Points $\boldsymbol{x}$ and $\widehat{\boldsymbol{x}}$ in spherical coordinates associated to a transversely isotropic material.

Assuming that the $x_{3}$-axis is the rotational symmetry axis of the material considered here, general expressions of the modulation functions (tensors) $\widehat{U}_{i k}, \widehat{U}_{i k ; j}$ and $\widehat{U}_{i k ; j \ell}$ for any $\boldsymbol{x}$, in terms of $\cos$ and $\sin$ functions of spherical angles $\phi$ and $\theta$ of $\boldsymbol{x}$, see Fig. 1, can be obtained from the expressions of these modulation functions for the corresponding point $\widehat{\boldsymbol{x}}$ by the following transformation of the components of these functions:

$$
\begin{aligned}
\widehat{U}_{i k}(\boldsymbol{x}) & =\Omega_{i a} \Omega_{k b} \widehat{U}_{a b}(\widehat{\boldsymbol{x}}), \\
\widehat{U}_{i k ; j}(\boldsymbol{x}) & =\Omega_{i a} \Omega_{k b} \Omega_{j c} \widehat{U}_{a b ; c}(\widehat{\boldsymbol{x}}), \\
\widehat{U}_{i k ; j \ell}(\boldsymbol{x}) & =\Omega_{i a} \Omega_{k b} \Omega_{j c} \Omega_{\ell d} \widehat{U}_{a b ; c d}(\widehat{\boldsymbol{x}}),
\end{aligned}
$$


where the rotation matrix $\Omega$ is defined as

$$
\boldsymbol{\Omega}(\theta)=\left(\begin{array}{ccc}
\cos \theta & -\sin \theta & 0 \\
\sin \theta & \cos \theta & 0 \\
0 & 0 & 1
\end{array}\right),
$$

the angle $\theta, 0 \leq \theta<2 \pi$, being shown in Fig. 1 .

Hence, if expressions of the modulation functions for a point $\widehat{x}$ (situated on the symmetry plane $x_{2}=0$ ) suitable for an efficient computer implementation are available, then it can be expected that the above transformation rules will lead to an efficient computer implementation of these modulation functions for any general point $\boldsymbol{x}$ as well. As will be shown in what follows this is the case of transversely isotropic materials with the $x_{3}$-axis being rotational symmetry axis.

It should be stressed that the transformation rules in (12-14) do not hold for general anisotropic materials (or any other orientation of transversely isotropic materials) as these rules are exclusively associated to the above mentioned rotational symmetry of the material with respect to the $x_{3}$-axis.

With reference to the plane $x_{2}=0$ (i.e. $\theta=0$ ) it will be useful to evaluate the particular expressions of $\boldsymbol{\Omega}(\theta)$ and its derivatives with respect to $\theta$ at $\theta=0$,

$$
\begin{gathered}
\boldsymbol{\Omega}(0)=\left(\begin{array}{lll}
1 & 0 & 0 \\
0 & 1 & 0 \\
0 & 0 & 1
\end{array}\right), \quad \boldsymbol{\Omega}^{\prime}(0)=\left(\begin{array}{ccc}
0 & -1 & 0 \\
1 & 0 & 0 \\
0 & 0 & 0
\end{array}\right), \\
\boldsymbol{\Omega}^{\prime \prime}(0)=\left(\begin{array}{ccc}
-1 & 0 & 0 \\
0 & -1 & 0 \\
0 & 0 & 0
\end{array}\right) .
\end{gathered}
$$

The expressions of the derivatives with respect to cartesian coordinates of a modulation function $f(\theta, \phi)$ for $\theta=0$ take the simple form:

$$
f_{, 1}=r^{-1} c \partial_{\phi} f, \quad f_{, 2}=r^{-1} s^{-1} \partial_{\theta} f, \quad f_{, 3}=-r^{-1} s \partial_{\phi} f
$$

and

$$
\begin{array}{lll}
f_{, 11}=r^{-2}\left(c^{2} \partial_{\phi \phi} f-2 c s \partial_{\phi} f\right), & f_{, 22}=r^{-2}\left(s^{-2} \partial_{\theta \theta} f+c s^{-1} \partial_{\phi} f\right), \\
f_{, 33}=r^{-2}\left(s^{2} \partial_{\phi \phi} f+2 c s \partial_{\phi} f\right), & f_{, 12}=r^{-2}\left(c s^{-1} \partial_{\phi \theta} f-s^{-2} \partial_{\theta} f\right), \\
f_{, 13}=r^{-2}\left(-c s \partial_{\phi \phi} f-\left(c^{2}-s^{2}\right) \partial_{\phi} f\right), & f_{, 23}=-r^{-2} \partial_{\phi \theta} f .
\end{array}
$$

The components of the unit vector $\widehat{x}$ are

\subsection{Modulation function $\widehat{U}_{i k}$}

$$
r_{, 1}=s, \quad r_{, 2}=0, \quad r_{3}=c .
$$

Explicit expressions for the non-zero terms of the modulation function $\widehat{U}_{i k}(\theta=0, \phi)=$ $H_{i k}(\theta=0, \phi)$ in the plane $x_{2}=0$, can be obtained as shown in [9], see also [1],

$$
\boldsymbol{H}(\widehat{\boldsymbol{x}})=\left(\begin{array}{ccc}
H_{11} & 0 & H_{13} \\
0 & H_{22} & 0 \\
H_{13} & 0 & H_{33}
\end{array}\right),
$$


where $H_{i k}=H_{i k}(0, \phi)$ are expressed as

$$
\begin{aligned}
& H_{11}=\frac{1}{C_{66} \beta_{3}}+\frac{C_{44} c^{2}+C_{33} s^{2}}{C_{11} C_{44} g h}-\frac{f}{\xi}, \\
& H_{22}=\frac{1}{C_{11} g}+\frac{f}{\xi}, \\
& H_{33}=\frac{1}{g h}\left\{\frac{h+c^{2}}{C_{44}}+\frac{s^{2}}{C_{11}}\right\}, \\
& H_{13}=\tilde{H}_{13} s,
\end{aligned}
$$

and

$$
\begin{aligned}
\tilde{H}_{13} & =\frac{\left(C_{13}+C_{44}\right) c}{C_{11} C_{44} g h}, \\
\beta_{3} & =\left\{\frac{C_{66} c^{2}+C_{44} s^{2}}{C_{66}}\right\}^{1 / 2}, \\
h & =\left\{c^{4}+\frac{\eta s^{2} c^{2}}{C_{11} C_{44}}+\frac{C_{33} s^{4}}{C_{11}}\right\}^{1 / 2}, \\
g & =\left\{2\left(h+c^{2}\right)+\frac{\eta s^{2}}{C_{11} C_{44}}\right\}^{1 / 2}, \\
\xi & =g\left(h+g \beta_{3}+\beta_{3}^{2}\right), \\
f & =\frac{h+c^{2}}{C_{66}}+\frac{g h}{C_{66} \beta_{3}}+\frac{C_{33} s^{2}}{C_{11} C_{44}}, \\
\eta & =C_{11} C_{33}-C_{13}^{2}-2 C_{13} C_{44},
\end{aligned}
$$

$\beta_{3}, h, g$ and $\xi$ being bounded and positive dimensionless functions given in terms of $c$ and $s$ [9]. This is a key issue for the expressions of modulation functions presented in the following sections, as these dimensionless functions are the only functions appearing in the denominators. This fact will guarantee the non-singular character of the modulation function expressions presented.

Finally, bringing together Equations (1), (4), (12), (15) and (20-23), an explicit and completely general expression for the fundamental solution $\boldsymbol{U}(\boldsymbol{x})$ in a transversely isotropic material is obtained. The form of this expression suitable for a computational implementation obtained by performing explicitly the transforms indicated in (12) is given in [1]. The presence of several zero components in $\boldsymbol{H}(\widehat{\boldsymbol{x}})$ and $\boldsymbol{\Omega}$ leads to simple and short expressions of the components of $\boldsymbol{H}(\boldsymbol{x})$.

\subsection{Modulation function $\widehat{U}_{i k ; j}$}

In order to evaluate $\widehat{U}_{i k ; j}(\theta=0, \phi)$, we first evaluate the derivatives indicated in (5). By applying formulas in (17) to (4), the following expressions of $\widehat{H}_{i k ; j}(\theta=0, \phi)$ defined 
in (7) are easily obtained in terms of derivatives of $H_{i k}(\theta, \phi)$ :

$$
\begin{aligned}
\widehat{H}_{i k ; 1} & =c \partial_{\phi} H_{i k}(0, \phi), \\
\widehat{H}_{i k ; 2} & =s^{-1} \partial_{\theta} H_{i k}(0, \phi), \\
\widehat{H}_{i k ; 3} & =-s \partial_{\phi} H_{i k}(0, \phi) .
\end{aligned}
$$

The required expressions of the first order partial derivatives of $H_{i k}(\theta, \phi)$ with respect to angles $\theta$ and $\phi$ can be evaluated from (12) in view of (4) as follows:

$$
\begin{aligned}
& \partial_{\phi} H_{i k}(\theta, \phi)=\Omega_{i m}(\theta) \Omega_{j n}(\theta) \partial_{\phi} H_{m n}(0, \phi) \\
& \partial_{\theta} H_{i k}(\theta, \phi)=\left(\Omega_{i m}^{\prime}(\theta) \Omega_{j n}(\theta)+\Omega_{i m}(\theta) \Omega_{j n}^{\prime}(\theta)\right) H_{m n}(0, \phi) .
\end{aligned}
$$

For the particular case of $\theta=0$, and in view of (16), derivatives (25) can be expressed as

$$
\begin{aligned}
\partial_{\phi} H_{i k}(0, \phi) & =\Omega_{i m}(0) \Omega_{j n}(0) \partial_{\phi} H_{m n}(0, \phi) \\
& =\left(\begin{array}{ccc}
H_{11}^{\prime} & 0 & H_{13}^{\prime} \\
0 & H_{22}^{\prime} & 0 \\
H_{13}^{\prime} & 0 & H_{33}^{\prime}
\end{array}\right),
\end{aligned}
$$

where $H_{m n}^{\prime}=\partial_{\phi} H_{m n}(\theta=0, \phi)$ represent the derivatives of the corresponding functions defined in (21) and will be expressed explicitly later on, and derivatives (26) as

$$
\begin{aligned}
\partial_{\theta} H_{i k}(0, \phi) & =\left(\Omega_{i m}^{\prime}(0) \Omega_{j n}(0)+\Omega_{i m}(0) \Omega_{j n}^{\prime}(0)\right) H_{m n}(0, \phi) \\
& =\left(\begin{array}{ccc}
0 & H_{11}-H_{22} & 0 \\
H_{11}-H_{22} & 0 & H_{13} \\
0 & H_{13} & 0
\end{array}\right)
\end{aligned}
$$

where $H_{m n}=H_{m n}(0, \phi)$.

The completely general and closed-form expressions of $\widehat{U}_{i k ; j}(\widehat{\boldsymbol{x}})$ can now be easily obtained by substitution of (24) with (27-28) and (19-20) into (9) considered for a point $\widehat{\boldsymbol{x}}($ i.e. $\theta=0$ ). The expressions obtained are presented in a compact form suitable for computer implementation:

$$
\begin{array}{lll}
\widehat{U}_{11 ; 1}=H_{11}^{\prime} c-H_{11} s, & \widehat{U}_{12 ; 2}=\tilde{H}_{12} s, & \widehat{U}_{11 ; 3}=-H_{11}^{\prime} s-H_{11} c, \\
\widehat{U}_{22 ; 1}=H_{22}^{\prime} c-H_{22} s, & \widehat{U}_{23 ; 2}=\tilde{H}_{13}, & \widehat{U}_{22 ; 3}=-H_{22}^{\prime} s-H_{22} c, \\
\widehat{U}_{33 ; 1}=H_{33}^{\prime} c-H_{33} s, & \widehat{U}_{33 ; 3}=-H_{33}^{\prime} s-H_{33} c, \\
\widehat{U}_{13 ; 1}=H_{13}^{\prime} c-H_{13} s, & \widehat{U}_{13 ; 3}=-H_{13}^{\prime} s-H_{13} c,
\end{array}
$$

where

$$
H_{11}^{\prime}=\tilde{H}_{11} s, \quad H_{22}^{\prime}=\tilde{H}_{22} s, \quad H_{33}^{\prime}=\tilde{H}_{33} s,
$$




$$
\begin{aligned}
\tilde{H}_{11} & =-\frac{\tilde{\beta}_{3}}{C_{66} \beta_{3}^{2}}-\frac{C_{44} c^{2}+C_{33} s^{2}}{C_{11} C_{44} g h}\left(\frac{\tilde{h}}{h}+\frac{\tilde{g}}{g}\right)+\frac{2\left(C_{33}-C_{44}\right) c}{C_{11} C_{44} g h}-\frac{1}{\xi}\left(\tilde{f}-\frac{\tilde{\xi} f}{\xi}\right) \\
\tilde{H}_{22} & =-\frac{\tilde{g}}{C_{11} g^{2}}+\frac{1}{\xi}\left(\tilde{f}-\frac{\tilde{\xi} f}{\xi}\right) \\
\tilde{H}_{33} & =-\frac{1}{g h}\left(\frac{2 c}{C_{11}}+\frac{\tilde{h}-2 c}{C_{44}}\right)-H_{33}\left(\frac{\tilde{h}}{h}+\frac{\tilde{g}}{g}\right) \\
\tilde{H}_{12} & =\frac{C_{33}}{C_{11} C_{44} g h}-\frac{\eta c^{2}+C_{33} C_{44} s^{2}}{C_{11} C_{44}\left(h+c^{2}\right)}\left(\frac{1}{C_{11} g h}+\frac{g}{C_{66} \beta_{3} \xi}\right) \\
& +\frac{1}{\xi}\left(\frac{\eta-2 C_{33} C_{66}}{C_{11} C_{44} C_{66}}+\frac{C_{44} g}{C_{66}^{2} \beta_{3}}\right) \\
H_{13}^{\prime} & =\frac{C_{13}+C_{44}}{C_{11} C_{44} g h}\left(c^{2}-s^{2}-\gamma c s\right) \\
\gamma & =\frac{g^{\prime}}{g}+\frac{h^{\prime}}{h}
\end{aligned}
$$

and

$$
\begin{aligned}
\tilde{\beta}_{3} & =\frac{\left(C_{44}-C_{66}\right) c}{C_{66} \beta_{3}}, \\
\tilde{h} & =\frac{1}{h}\left(\frac{\eta c}{C_{11} C_{44}}\left(c^{2}-s^{2}\right)+\frac{2 C_{33} c s^{2}}{C_{11}}-2 c^{3}\right), \\
\tilde{g} & =\frac{1}{g}\left(\frac{\eta c}{C_{11} C_{44}}+\tilde{h}-2 c\right), \\
\tilde{\xi} & =g\left(\frac{2\left(C_{44}-C_{66}\right) c}{C_{66}}+\tilde{h}+\beta_{3} \tilde{g}+\tilde{\beta}_{3} g\right)+\frac{\tilde{g} \xi}{g}, \\
\tilde{f} & =\frac{\tilde{h}-2 c}{C_{66}}+\frac{1}{C_{66} \beta_{3}}\left(h \tilde{g}+\tilde{h} g-\frac{\tilde{\beta}_{3} g h}{\beta_{3}}\right)+\frac{2 C_{33} c}{C_{11} C_{44}} .
\end{aligned}
$$

In (32), 'tilde' functions are related to $\beta_{3}^{\prime}, h^{\prime}, g^{\prime}, \xi^{\prime}$, and $f^{\prime}$, that represent the first order derivatives with respect to the angle $\phi$ (at $\theta=0$ ) of the corresponding functions defined in (22-23), in the following way:

$$
\beta_{3}^{\prime}=\tilde{\beta_{3}} s, \quad h^{\prime}=\tilde{h} s, \quad g^{\prime}=\tilde{g} s, \quad \xi^{\prime}=\tilde{\xi} s, \quad f^{\prime}=\tilde{f} s .
$$

'Tilde' functions are introduced in order to avoid the presence of divisions by $s$, that will lead eventually in divisions by zero when $\phi=0$.

The remaining components of $\widehat{U}_{i k ; j}(\widehat{\boldsymbol{x}})$ vanish,

$$
\widehat{U}_{12 ; 1}=\widehat{U}_{23 ; 1}=\widehat{U}_{11 ; 2}=\widehat{U}_{13 ; 2}=\widehat{U}_{22 ; 2}=\widehat{U}_{33 ; 2}=\widehat{U}_{12 ; 3}=\widehat{U}_{23 ; 3}=0 .
$$

It should be mentioned that in the original expression of $\widehat{U}_{12,2}(\widehat{\boldsymbol{x}})$, directly obtained by differentiating (24), the term $\left(H_{11}-H_{22}\right) / s$ appeared, which would lead to zero divided by zero for points at the $x_{3}$-axis. This term, which has a finite limit value for $\phi \rightarrow 0$ or $\pi$, has been rewritten in the form $\tilde{H}_{12} s$, which is well defined for any point with $r>0$. 


\subsection{Modulation function $\widehat{U}_{i k ; j \ell}$}

To determine $\widehat{U}_{i k ; j \ell}(\theta=0, \phi)$, we first evaluate the second order derivatives appearing in (6). Using formulas in (18) applied to (4), the following expressions of $\widehat{H}_{i k ; j \ell}(\theta=0, \phi)$ defined in (8) are easily obtained in terms of derivatives of $H_{i k}(\theta, \phi)$ :

$$
\begin{aligned}
\widehat{H}_{i k ; 11} & =c^{2} \partial_{\phi \phi}^{2} H_{i k}(0, \phi)-2 c s \partial_{\phi} H_{i k}(0, \phi), \\
\widehat{H}_{i k ; 22} & =s^{-2} \partial_{\theta \theta}^{2} H_{i k}(0, \phi)+c s^{-1} \partial_{\phi} H_{i k}(0, \phi), \\
\widehat{H}_{i k ; 33} & =s^{2} \partial_{\phi \phi}^{2} H_{i k}(0, \phi)+2 c s \partial_{\phi} H_{i k}(0, \phi), \\
\widehat{H}_{i k ; 12} & =c s^{-1} \partial_{\phi \theta}^{2} H_{i k}(0, \phi)-s^{-2} \partial_{\theta} H_{i k}(0, \phi), \\
\widehat{H}_{i k ; 13} & =-c s \partial_{\phi \phi}^{2} H_{i k}(0, \phi)-\left(c^{2}-s^{2}\right) \partial_{\phi} H_{i k}(0, \phi), \\
\widehat{H}_{i k ; 23} & =-\partial_{\phi \theta}^{2} H_{i k}(0, \phi) .
\end{aligned}
$$

Recall the following symmetry relations, due to the interchanging the order of taking partial derivatives,

$$
\widehat{H}_{i k ; j \ell}=\widehat{H}_{i k ; \ell j} .
$$

The required expressions of the second order partial derivatives of $H_{i k}(\theta, \phi)$ with respect to angles $\theta$ and $\phi$ can be evaluated from (12) in view of (4) as follows:

$$
\begin{aligned}
\partial_{\phi \phi}^{2} H_{i k}(\theta, \phi) & =\Omega_{i m}(\theta) \Omega_{j n}(\theta) \partial_{\phi \phi} H_{m n}(0, \phi) \\
\partial_{\phi \theta}^{2} H_{i k}(\theta, \phi) & =\left(\Omega_{i m}^{\prime}(\theta) \Omega_{j n}(\theta)+\Omega_{i m}(\theta) \Omega_{j n}^{\prime}(\theta)\right) \partial_{\phi} H_{m n}(0, \phi) \\
\partial_{\theta \theta}^{2} H_{i k}(\theta, \phi) & =\left(\Omega_{i m}^{\prime \prime}(\theta) \Omega_{j n}(\theta)+2 \Omega_{i m}^{\prime}(\theta) \Omega_{j n}^{\prime}(\theta)+\Omega_{i m}(\theta) \Omega_{j n}^{\prime \prime}(\theta)\right) H_{m n}(0, \phi) .
\end{aligned}
$$

For the particular case of $\theta=0$, and in view of (16), derivatives (37-39) can be expressed as:

$$
\begin{gathered}
\partial_{\phi \phi}^{2} H_{i k}(0, \phi)=\Omega_{i m}(0) \Omega_{j n}(0) \partial_{\phi \phi} H_{m n}(0, \phi) \\
=\left(\begin{array}{ccc}
H_{11}^{\prime \prime} & 0 & H_{13}^{\prime \prime} \\
0 & H_{22}^{\prime \prime} & 0 \\
H_{13}^{\prime \prime} & 0 & H_{33}^{\prime \prime}
\end{array}\right) \\
\partial_{\phi \theta}^{2} H_{i k}(0, \phi)=\left(\Omega_{i m}^{\prime}(0) \Omega_{j n}(0)+\Omega_{i m}(0) \Omega_{j n}^{\prime}(0)\right) \partial_{\phi} H_{m n}(0, \phi) \\
=\left(\begin{array}{ccc}
H_{11}^{\prime}-H_{22}^{\prime} & H_{11}^{\prime}-H_{22}^{\prime} & 0 \\
0 & 0 & H_{13}^{\prime}
\end{array}\right) \\
\partial_{\theta \theta}^{2} H_{i k}(0, \phi)=\left(\begin{array}{ccc}
\left.\Omega_{i m}^{\prime \prime}(0) \Omega_{j n}(0)+2 \Omega_{i m}^{\prime}(0) \Omega_{j n}^{\prime}(0)+\Omega_{i m}(0) \Omega_{j n}^{\prime \prime}(0)\right) H_{m n}(0, \phi) \\
0 & 0 & -H_{13} \\
-2\left(H_{11}-H_{22}\right) & 2\left(H_{11}-H_{22}\right) & 0 \\
-H_{13} & 0 & 0
\end{array}\right)
\end{gathered}
$$


where $H_{m n}^{\prime \prime}=\partial_{\phi \phi}^{2} H_{m n}(\theta=0, \phi)$ represent the second order derivatives of the corresponding functions defined in (21) and will be expressed explicitly in the following.

The completely general and closed-form expressions of $\widehat{U}_{i k ; j \ell}(\widehat{\boldsymbol{x}})$ can now be easily obtained by substitution of (35), (24) and (20) into (10) considered for a point $\widehat{\boldsymbol{x}}$. The $\widehat{U}_{i k ; j \ell}$ expressions obtained take the form:

$$
\begin{aligned}
& \widehat{U}_{11 ; 11}=H_{11}^{\prime \prime} c^{2}-4 H_{11}^{\prime} c s-H_{11} c^{2}+2 H_{11} s^{2}, \\
& \widehat{U}_{11 ; 13}=-H_{11}^{\prime \prime} c s-2 H_{11}^{\prime}\left(c^{2}-s^{2}\right)+3 H_{11} c s, \\
& \widehat{U}_{11 ; 22}=\tilde{H}_{11} c-2 \tilde{H}_{12}-H_{11}, \\
& \widehat{U}_{11 ; 33}=H_{11}^{\prime \prime} s^{2}+4 H_{11}^{\prime} c s+2 H_{11} c^{2}-H_{11} s^{2}, \\
& \widehat{U}_{12 ; 12}=\left(\tilde{H}_{11}-\tilde{H}_{22}\right) c-\tilde{H}_{12}-H_{11}+H_{22}, \\
& \widehat{U}_{12 ; 23}=-H_{11}^{\prime}+H_{22}^{\prime}-\tilde{H}_{12} c s, \\
& \widehat{U}_{13 ; 11}=H_{13}^{\prime \prime} c^{2}-4 H_{13}^{\prime} c s-H_{13} c^{2}+2 H_{13} s^{2}, \\
& \widehat{U}_{13 ; 13}=-H_{13}^{\prime \prime} c s-2 H_{13}^{\prime}\left(c^{2}-s^{2}\right)+3 H_{13} c s, \\
& \widehat{U}_{13 ; 22}=-\tilde{H}_{13}(2 s+\gamma c)-H_{13}, \\
& \widehat{U}_{13 ; 33}=H_{13}^{\prime \prime} s^{2}+4 H_{13}^{\prime} c s+2 H_{13} c^{2}-H_{13} s^{2}, \\
& \widehat{U}_{22 ; 11}=H_{22}^{\prime \prime} c^{2}-4 H_{22}^{\prime} c s-H_{22} c^{2}+2 H_{22} s^{2}, \\
& \widehat{U}_{22 ; 13}=-H_{22}^{\prime \prime} c s-2 H_{22}^{\prime}\left(c^{2}-s^{2}\right)+3 H_{22} c s, \\
& \widehat{U}_{22 ; 22}=\tilde{H}_{22} c+2 \tilde{H}_{12}-H_{22}, \\
& \widehat{U}_{22 ; 33}=H_{22}^{\prime \prime} s^{2}+4 H_{22}^{\prime} c s+2 H_{22} c^{2}-H_{22} s^{2}, \\
& \widehat{U}_{23 ; 23}=-H_{13}^{\prime}-\tilde{H}_{13} c, \\
& \widehat{U}_{33 ; 11}=H_{33}^{\prime \prime} c^{2}-4 H_{33}^{\prime} c s-H_{33} c^{2}+2 H_{33} s^{2}, \\
& \widehat{U}_{33 ; 13}=-H_{33}^{\prime \prime} c s-2 H_{33}^{\prime}\left(c^{2}-s^{2}\right)+3 H_{33} c s, \\
& \widehat{U}_{33 ; 22}=-H_{33}+\tilde{H}_{33} c, \\
& \widehat{U}_{33 ; 33}=H_{33}^{\prime \prime} s^{2}+4 H_{33}^{\prime} c s+2 H_{33} c^{2}-H_{33} s^{2},
\end{aligned}
$$

and

$$
\widehat{U}_{23,12}=\widehat{U}_{13,22},
$$

where 


$$
\begin{aligned}
& H_{13}^{\prime \prime}=\frac{C_{13}+C_{44}}{C_{11} C_{44} h g}\left[\left(\frac{g^{\prime 2}}{g^{2}}+\frac{h^{\prime 2}}{h^{2}}-4\right) c s-\frac{g^{\prime \prime} c s+g^{\prime}\left(c^{2}-s^{2}\right)}{g}\right. \\
& \left.-\frac{h^{\prime \prime} c s+h^{\prime}\left(c^{2}-s^{2}\right)}{h}\right]-H_{13} \gamma, \\
& H_{33}^{\prime \prime}=-H_{33}\left(\frac{g^{\prime \prime} g-g^{\prime 2}}{g^{2}}+\frac{h^{\prime \prime} h-h^{\prime 2}}{h^{2}}\right)-H_{33}^{\prime} \gamma-\frac{\gamma}{g h}\left(\frac{h^{\prime}-2 c s}{C_{44}}+\frac{2 c s}{C_{11}}\right) \\
& +\frac{1}{g h}\left(\frac{h^{\prime \prime}-2\left(c^{2}-s^{2}\right)}{C_{44}}+\frac{2\left(c^{2}-s^{2}\right)}{C_{11}}\right), \\
& H_{22}^{\prime \prime}=\frac{2 g^{\prime 2}-g^{\prime \prime} g}{C_{11} g^{3}}+\frac{1}{\xi}\left(f^{\prime \prime}-\frac{\left(\xi^{\prime \prime} f+\xi^{\prime} f^{\prime}\right) \xi-f \xi^{2}}{\xi^{2}}\right)+\frac{\xi^{\prime}}{\xi^{2}}\left(\frac{f \xi^{\prime}}{\xi}-f^{\prime}\right) \text {, } \\
& H_{11}^{\prime \prime}=\frac{2 \beta_{3}^{\prime 2}-\beta_{3}^{\prime \prime} \beta_{3}}{C_{66} \beta_{3}^{3}}+\frac{C_{44} c^{2}+C_{33} s^{2}}{C_{11} C_{44} g h}\left(\gamma^{2}-\frac{g^{\prime \prime} g-g^{\prime 2}}{g^{2}}-\frac{h^{\prime \prime} h-h^{\prime 2}}{h^{2}}\right) \\
& -\frac{4\left(C_{33}-C_{44}\right) c s \gamma}{C_{11} C_{44} g h}-\frac{1}{\xi}\left(f^{\prime \prime}-\frac{\left(\xi^{\prime \prime} f+\xi^{\prime} f^{\prime}\right) \xi-f \xi^{\prime 2}}{\xi^{2}}\right)-\frac{\xi^{\prime}}{\xi^{2}}\left(\frac{f \xi^{\prime}}{\xi}-f^{\prime}\right) \\
& +\frac{2\left(C_{33}-C_{44}\right)\left(c^{2}-s^{2}\right)}{C_{11} C_{44} g h}, \\
& \beta_{3}^{\prime \prime}=-\frac{\beta_{3}^{\prime 2}}{\beta_{3}}+\frac{\left(C_{44}-C_{66}\right)}{C_{66} \beta_{3}}\left(c^{2}-s^{2}\right), \\
& h^{\prime \prime}=\frac{1}{h}\left(-h^{\prime 2}-2 h^{2}+2\left(3+\frac{3 C_{33}}{C_{11}}-\frac{\eta}{C_{11} C_{44}}\right) c^{2} s^{2}+\frac{\eta\left(c^{2}-s^{2}\right)^{2}}{C_{11} C_{44}}\right), \\
& g^{\prime \prime}=\frac{1}{g}\left(h^{\prime \prime}-g^{\prime 2}+\left(\frac{\eta}{C_{11} C_{44}}-2\right)\left(c^{2}-s^{2}\right)\right) \text {, } \\
& \xi^{\prime \prime}=g\left(h^{\prime \prime}+\beta_{3} g^{\prime \prime}+\beta_{3}^{\prime \prime} g+2 \beta_{3}^{\prime} g^{\prime}+\frac{2\left(C_{44}-C_{66}\right)\left(c^{2}-s^{2}\right)}{C_{66}}\right) \\
& +\frac{g^{\prime \prime} \xi}{g}+2 g^{\prime}\left(\frac{\xi^{\prime}}{g}-\frac{g^{\prime} \xi}{g^{2}}\right), \\
& f^{\prime \prime}=\frac{1}{C_{66} \beta_{3}}\left(h^{\prime \prime} g+h g^{\prime \prime}-\frac{\beta_{3}^{\prime \prime} g h+\beta_{3}^{\prime} g^{\prime} h+\beta_{3}^{\prime} h^{\prime} g}{\beta_{3}}+\frac{2 g h \beta_{3}^{\prime 2}}{\beta_{3}^{2}}+2 h^{\prime} g^{\prime}\right) \\
& -\frac{\left(h g^{\prime}+h^{\prime} g\right) \beta_{3}^{\prime}}{C_{66} \beta_{3}^{2}}+\frac{2 C_{33}\left(c^{2}-s^{2}\right)}{C_{11} C_{44}}+\frac{h^{\prime \prime}-2\left(c^{2}-s^{2}\right)}{C_{66}} .
\end{aligned}
$$

Functions $\beta_{3}^{\prime \prime}, h^{\prime \prime}, g^{\prime \prime}, \xi^{\prime \prime}$ and $f^{\prime \prime}$ represent the second order derivatives with respect to the angle $\phi$ of the corresponding functions defined in (22-23).

The remaining components of $\widehat{U}_{i k ; j l}(\widehat{\boldsymbol{x}})$ vanish,

$$
\begin{aligned}
& \widehat{U}_{11 ; 12}=\widehat{U}_{11 ; 23}=\widehat{U}_{12 ; 11}=\widehat{U}_{12 ; 13}=\widehat{U}_{12 ; 22}=\widehat{U}_{12 ; 33}=\widehat{U}_{13 ; 12}=\widehat{U}_{13 ; 23}=0 \\
& \widehat{U}_{22 ; 12}=\widehat{U}_{22 ; 23}=\widehat{U}_{23 ; 11}=\widehat{U}_{23 ; 13}=\widehat{U}_{23 ; 22}=\widehat{U}_{23 ; 33}=\widehat{U}_{33 ; 12}=\widehat{U}_{33 ; 23}=0 .
\end{aligned}
$$




\section{Final Remarks}

The above closed-form expressions of the fundamental solution and its derivatives can be directly used to deduce expressions of the boundary integral kernels $T_{i k}, D_{i j k}$ and $S_{i j k}$ in the Somigliana displacement and stress identities [24, 25, 26], after application of the constitutive law, as shown by Távara et. al. [1, 2]. Explicit expressions of these kernels suitable for simple and efficient computer implementation are presented, and their correctness tested on some examples with known analytic solutions in Távara et. al. $[1,2]$ An advanced application was also introduced in [2]. Some plots of several terms of the fundamental solution and its derivatives are presented in Appendix A.

The main advantage of the present formulation is that unique expressions in real variable covering all orientations of the transversely isotropic materials and all combinations of materials parameters are obtained. These features have not even achieved by any other previous expressions to the best knowledge of the authors.

Additionally, these expressions seem to be more efficient, concerning the computational time, than previous expressions, which have also been implemented in our 3-D BEM code for the purpose of comparison. One of the reason for this efficiency consists of the relatively large number number of vanishing components of the modulation functions for $\widehat{\boldsymbol{x}}$ : 2 terms from 6 for $\widehat{U}_{i k}(\widehat{\boldsymbol{x}}), 8$ terms from 18 for $\widehat{U}_{i k ; j}(\widehat{\boldsymbol{x}})$, and 16 terms from

36 for $\widehat{U}_{i k ; j \ell}(\widehat{\boldsymbol{x}})$. It should be noticed that a Fortran subroutine containing all the above mentioned expressions is presented in Appendix B.

\section{APPENDIX A: GRAPHICAL REPRESENTATION OF SOME TERMS OF THE FUNDAMENTAL SOLUTION AND ITS DERIVATIVES}

The properties of the fundamental solution and its derivatives presented in the present work are illustrated in the following Figures. Some terms are plotted for the 3 different cases of material combinations, $\triangle=\sqrt{C_{11} C_{33}}-C_{13}-2 C_{44}$ (positive, negative or zero). The material properties used are the ones presented in Table 1 in [1]. The plots were done by means of the computer software Mathematica Version 8.0. 


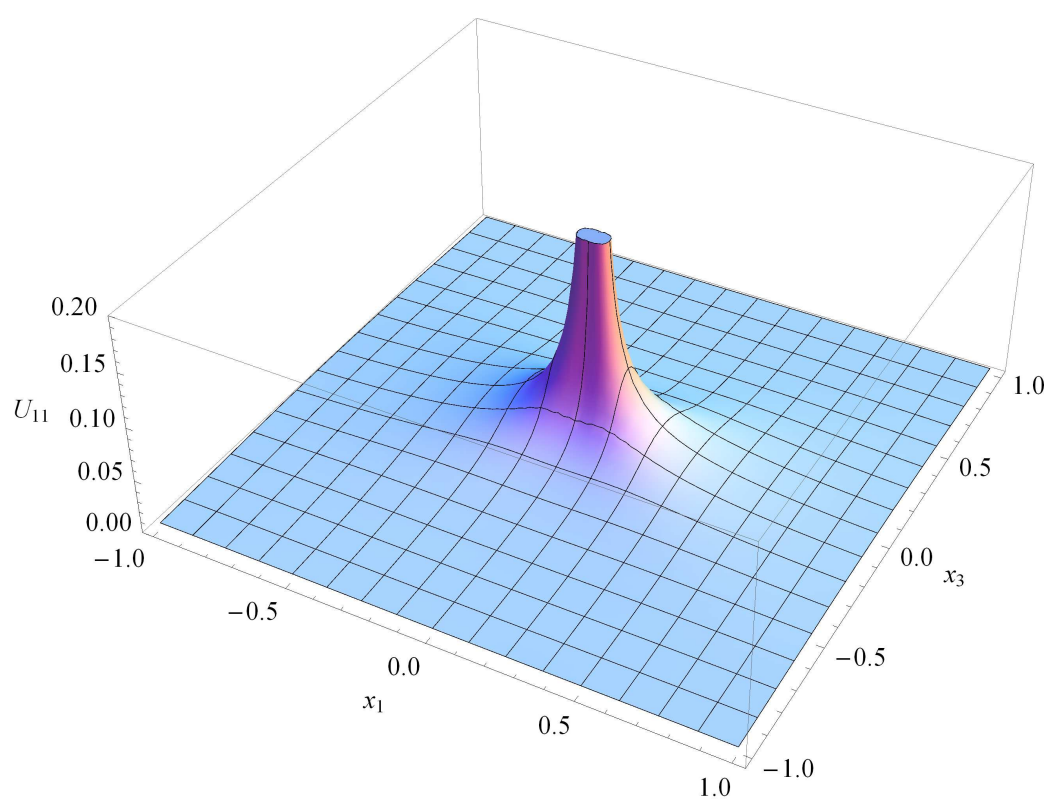

Figure 2: $U_{11}$ in plane $x_{2}=0$, for $\triangle<0$.

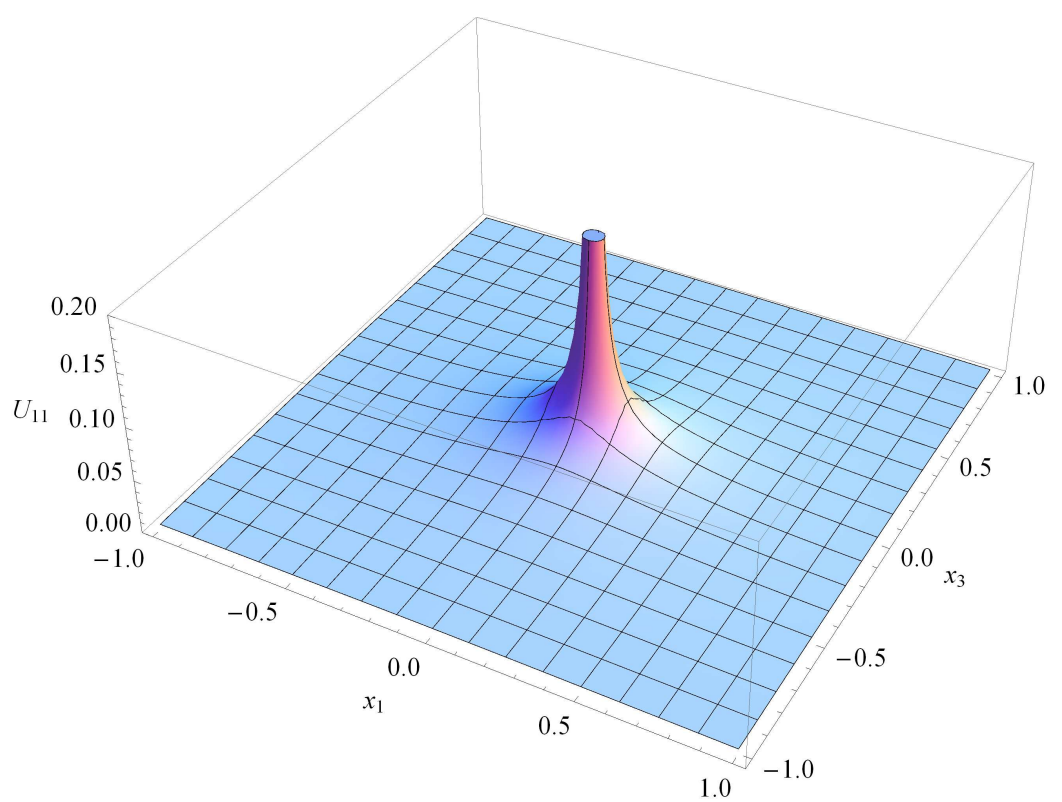

Figure 3: $U_{11}$ in plane $x_{2}=0$, for $\triangle=0$. 


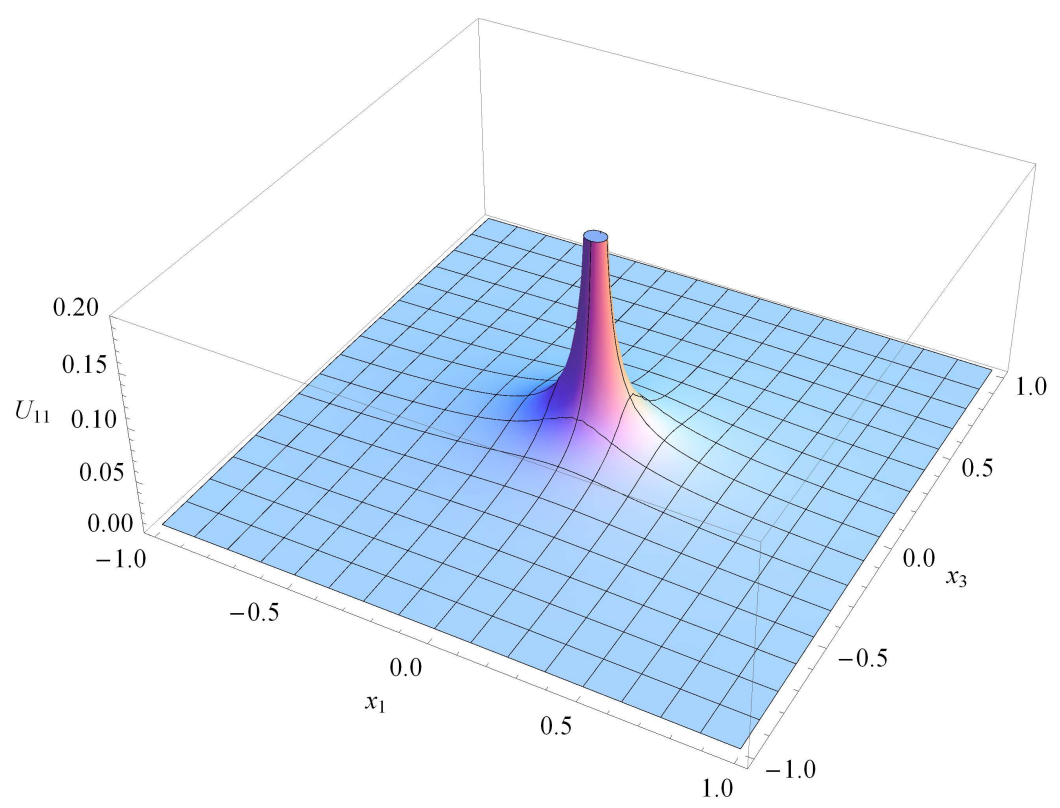

Figure 4: $U_{11}$ in plane $x_{2}=0$, for $\triangle>0$.

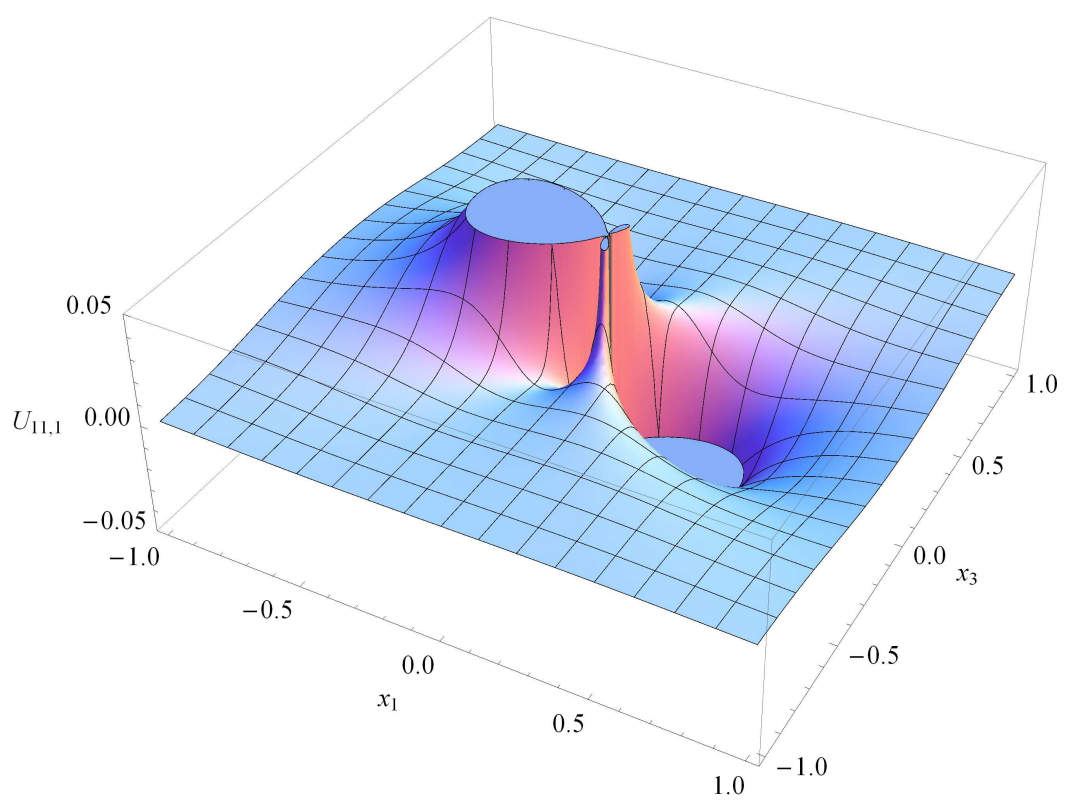

Figure 5: $U_{11,1}$ in plane $x_{2}=0$, for $\triangle<0$. 


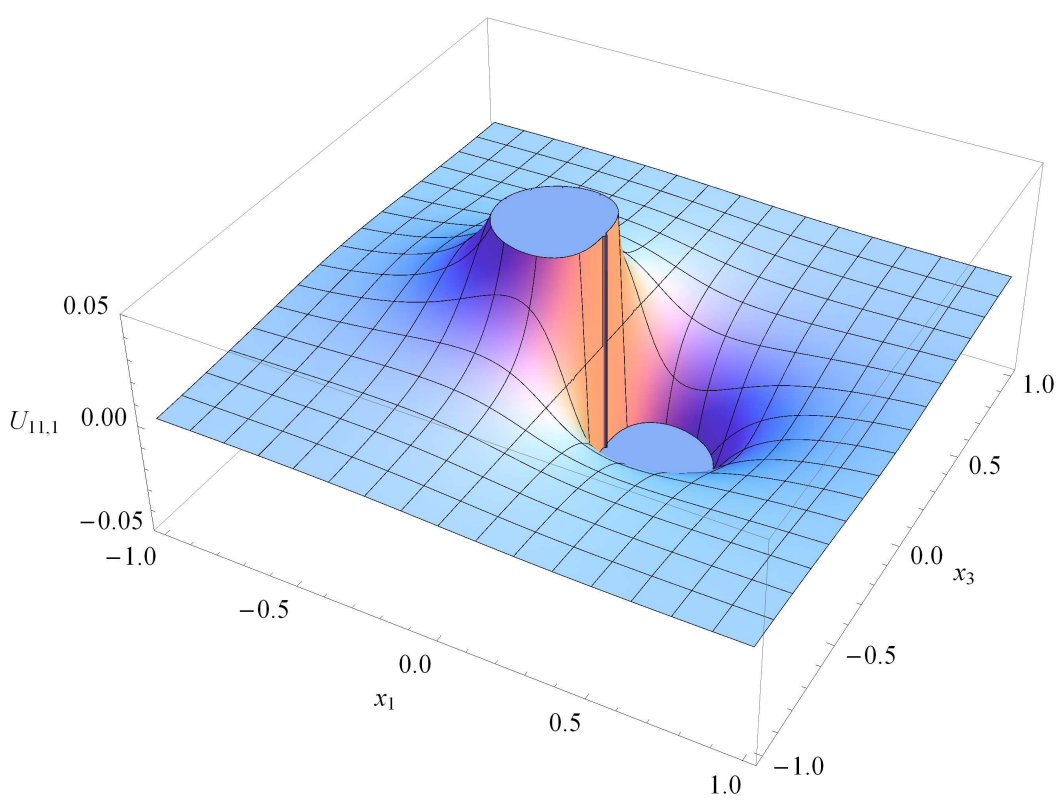

Figure 6: $U_{11,1}$ in plane $x_{2}=0$, for $\triangle=0$.

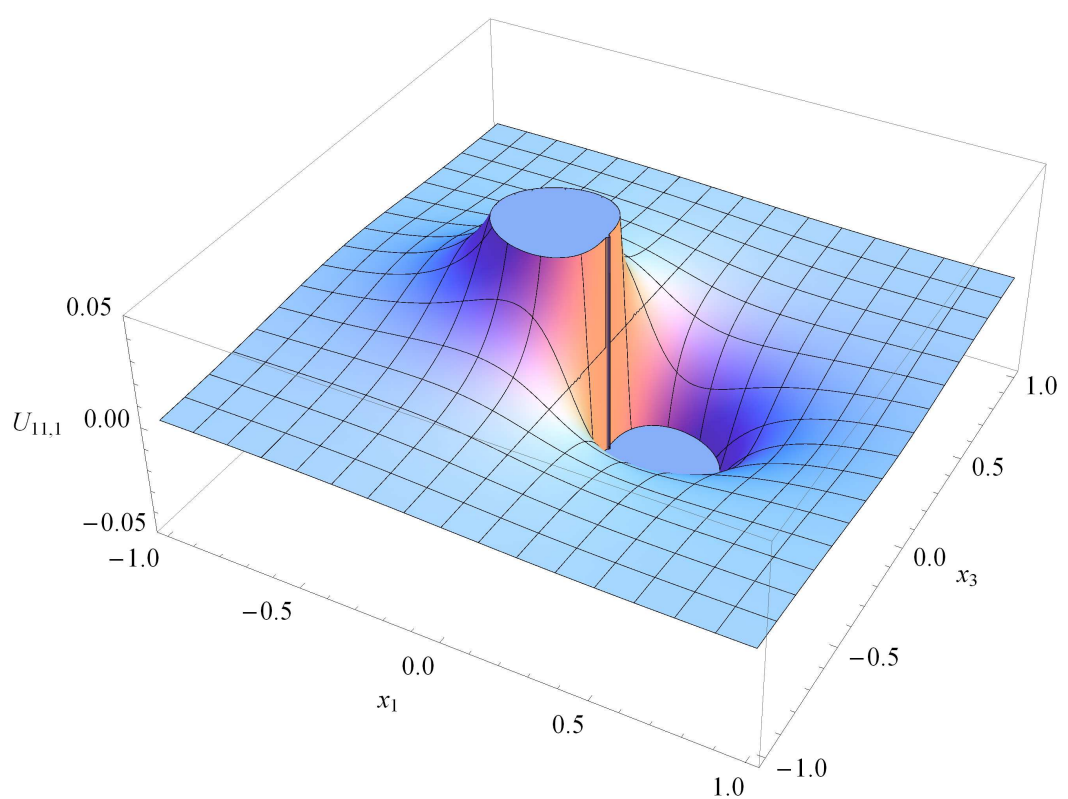

Figure 7: $U_{11,1}$ in plane $x_{2}=0$, for $\triangle>0$. 


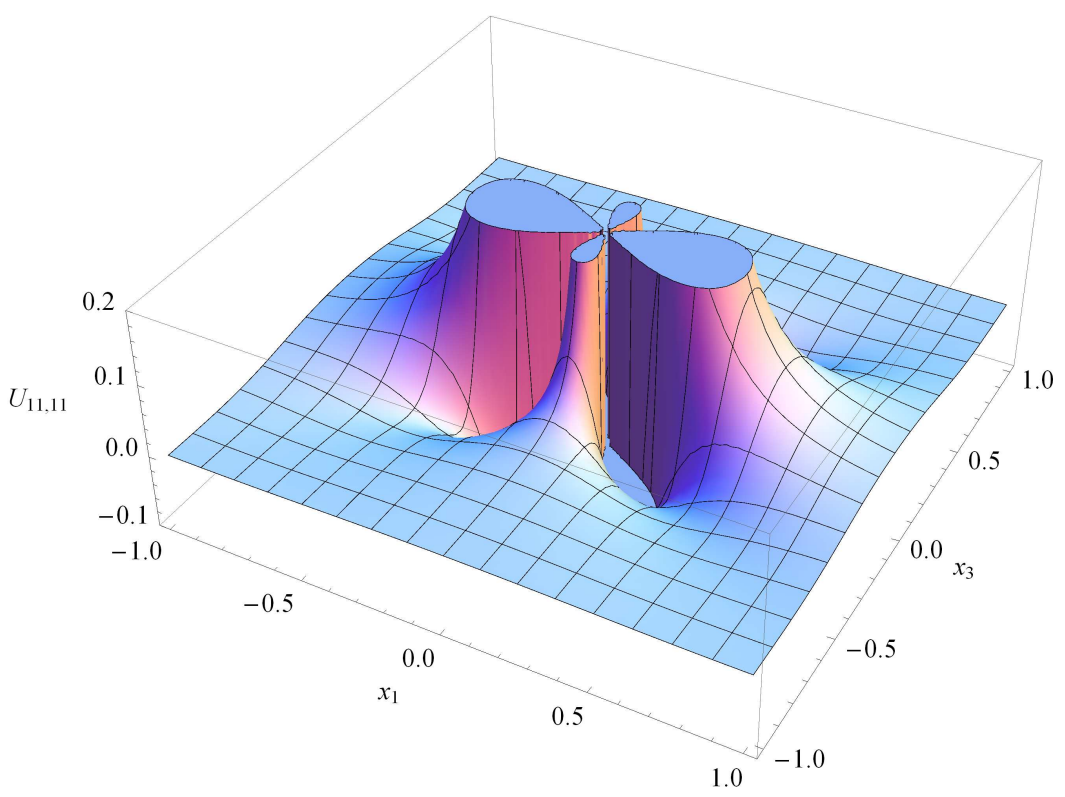

Figure 8: $U_{11,11}$ in plane $x_{2}=0$, for $\triangle<0$.

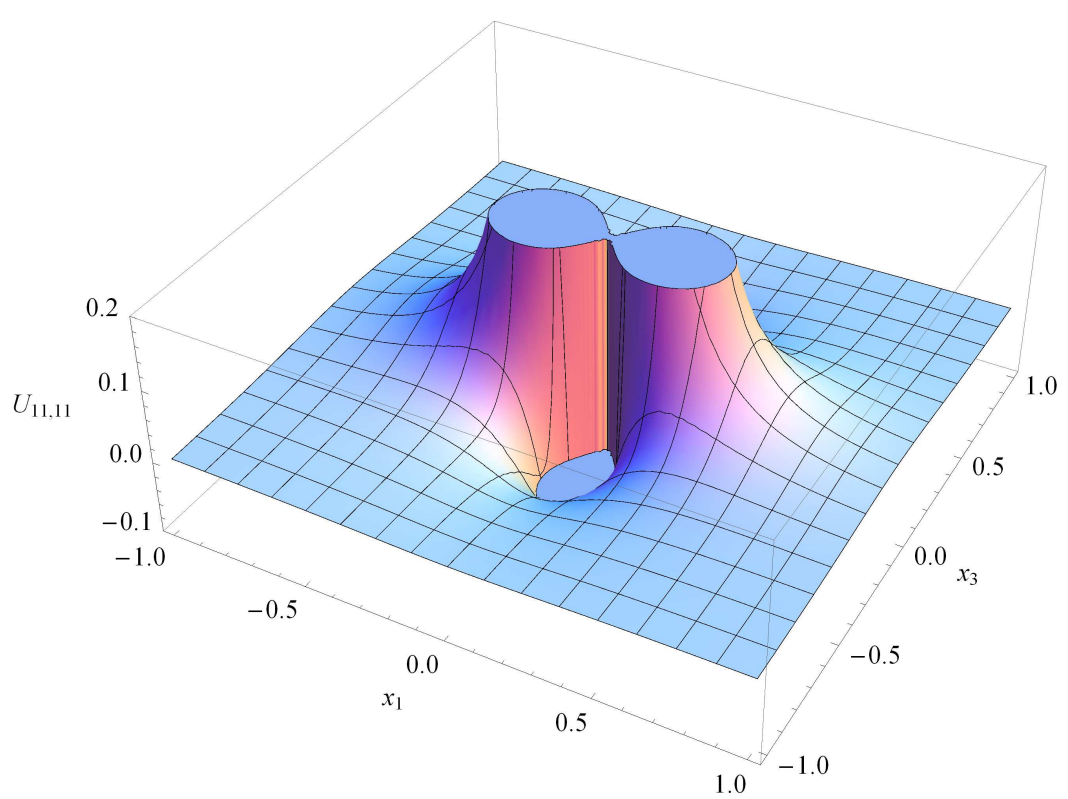

Figure 9: $U_{11,11}$ in plane $x_{2}=0$, for $\triangle=0$. 


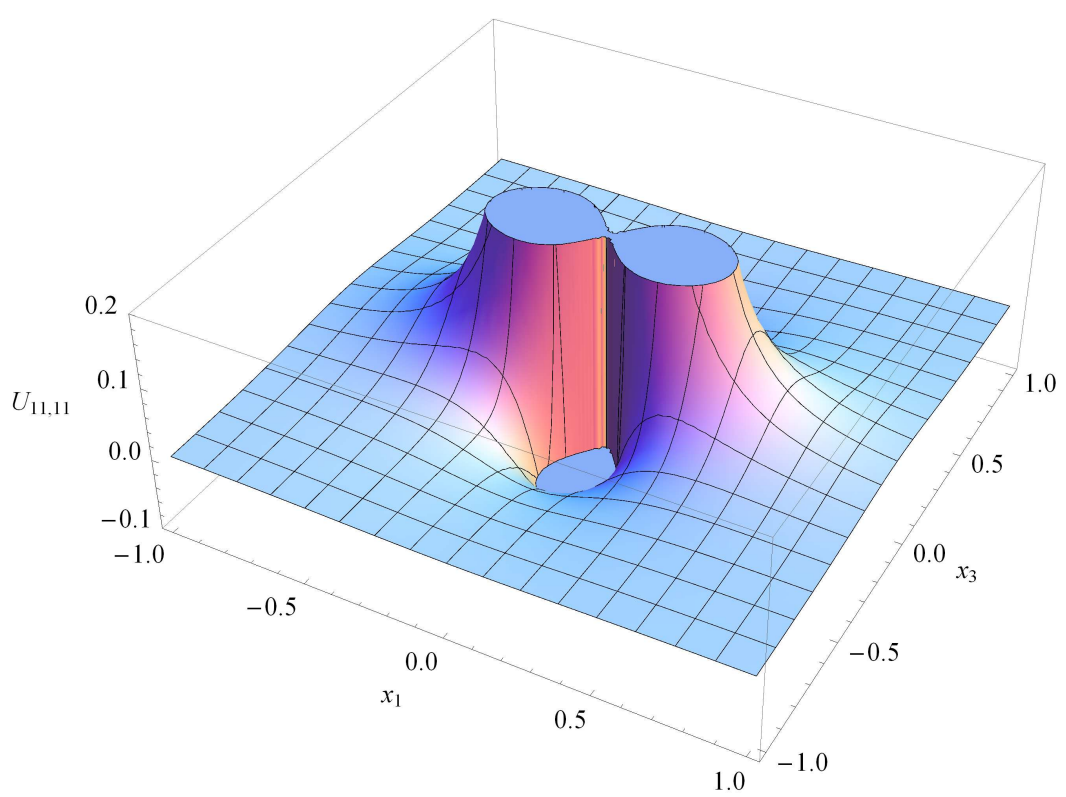

Figure 10: $U_{11,11}$ in plane $x_{2}=0$, for $\triangle>0$.

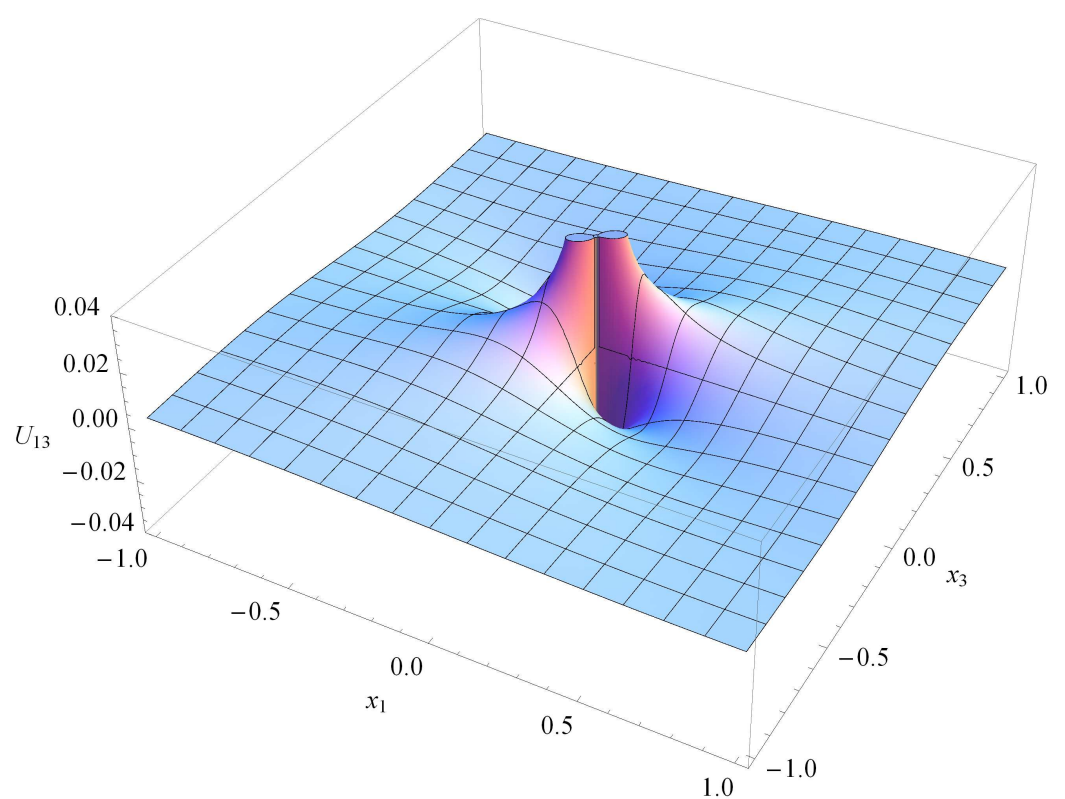

Figure 11: $U_{13}$ in plane $x_{2}=0$, for $\triangle<0$. 


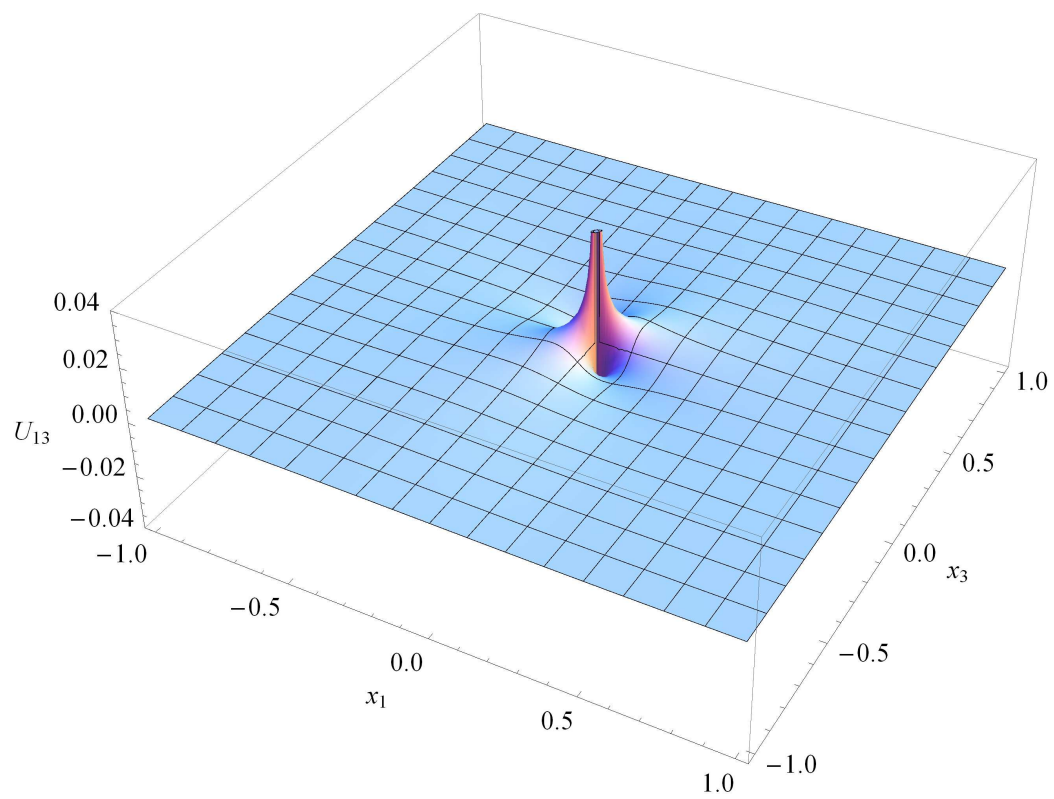

Figure 12: $U_{13}$ in plane $x_{2}=0$, for $\triangle=0$.

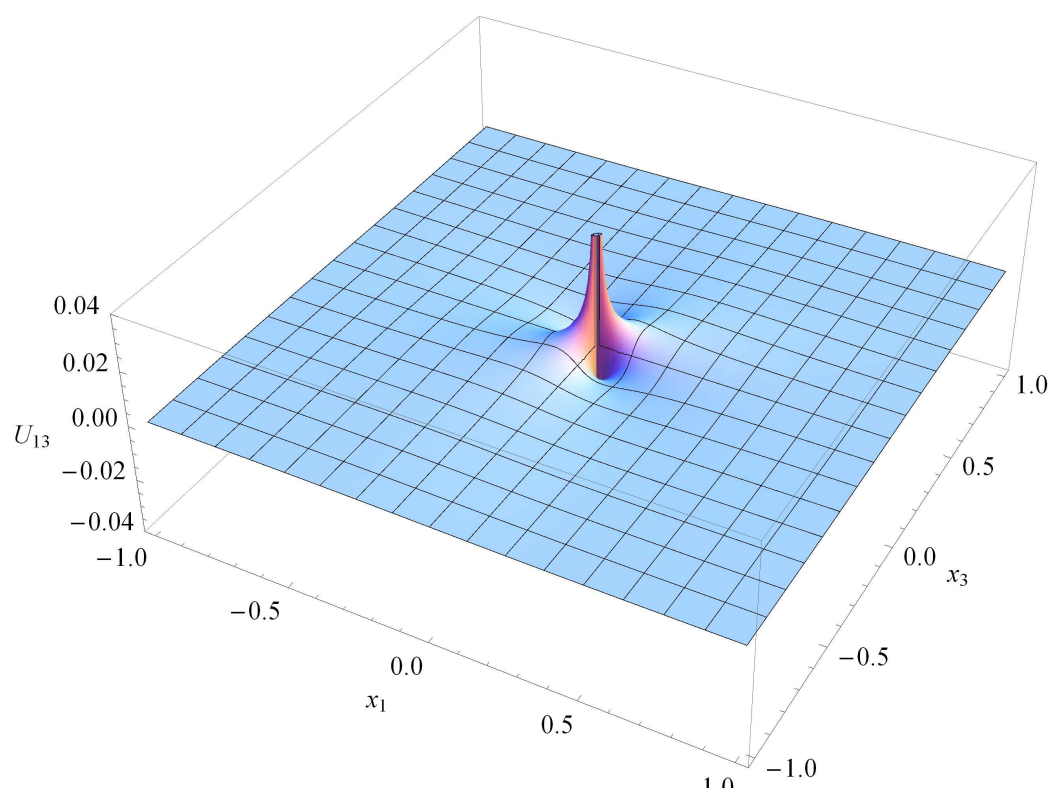

Figure 13: $U_{13}$ in plane $x_{2}=0$, for $\triangle>0$. 


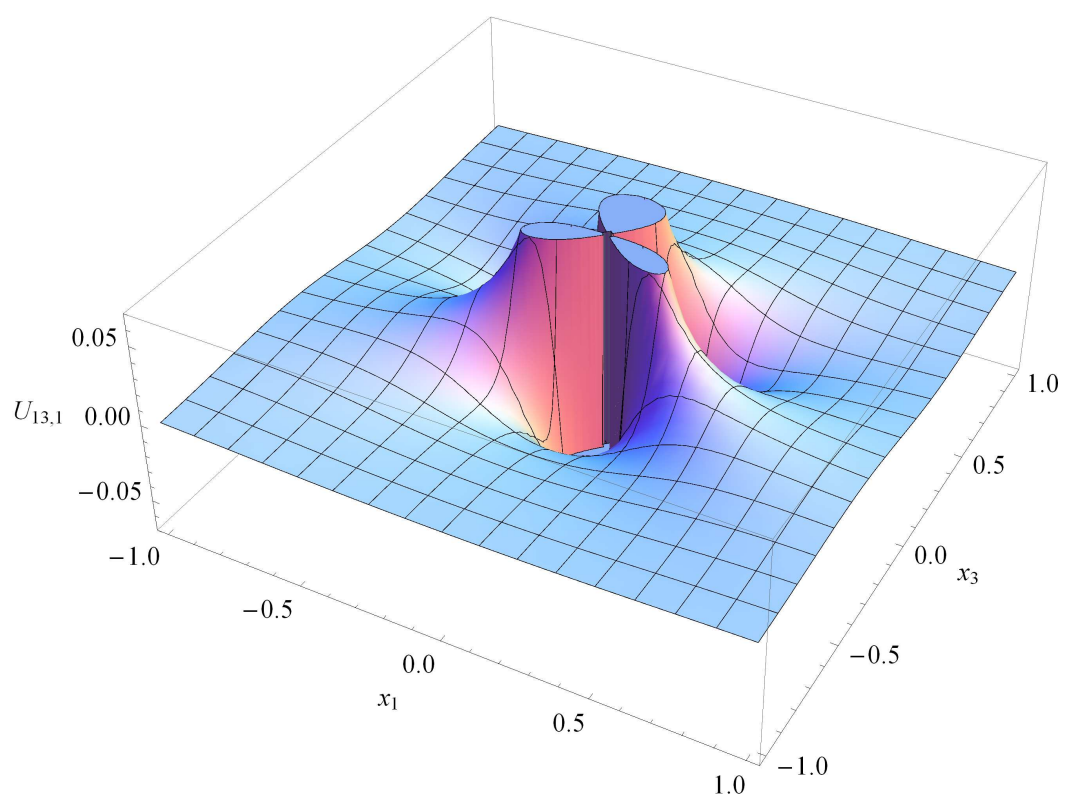

Figure 14: $U_{13,1}$ in plane $x_{2}=0$, for $\triangle<0$.

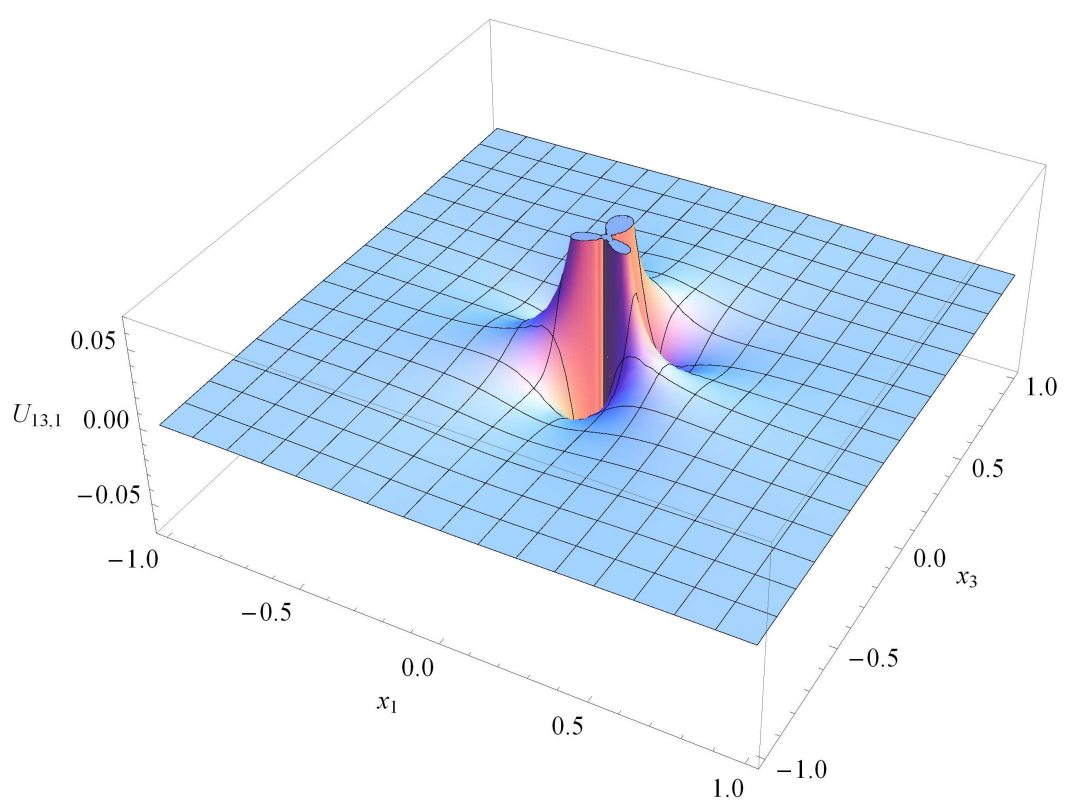

Figure 15: $U_{13,1}$ in plane $x_{2}=0$, for $\triangle=0$. 


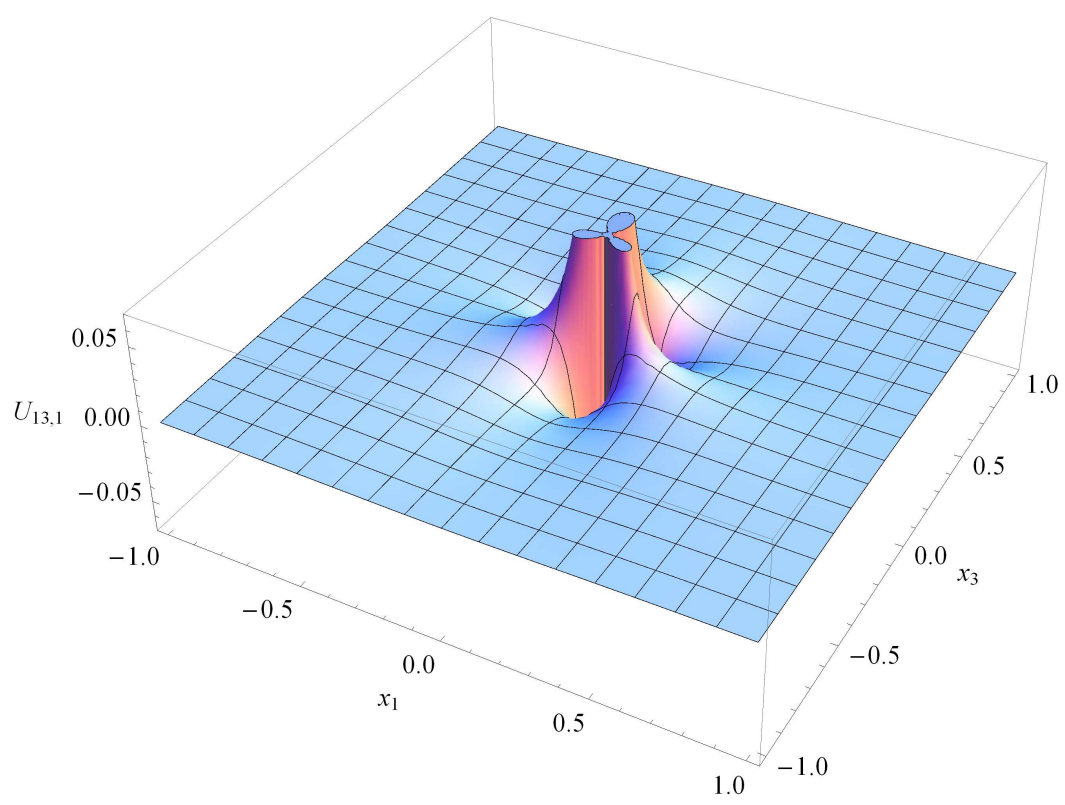

Figure 16: $U_{13,1}$ in plane $x_{2}=0$, for $\triangle>0$.

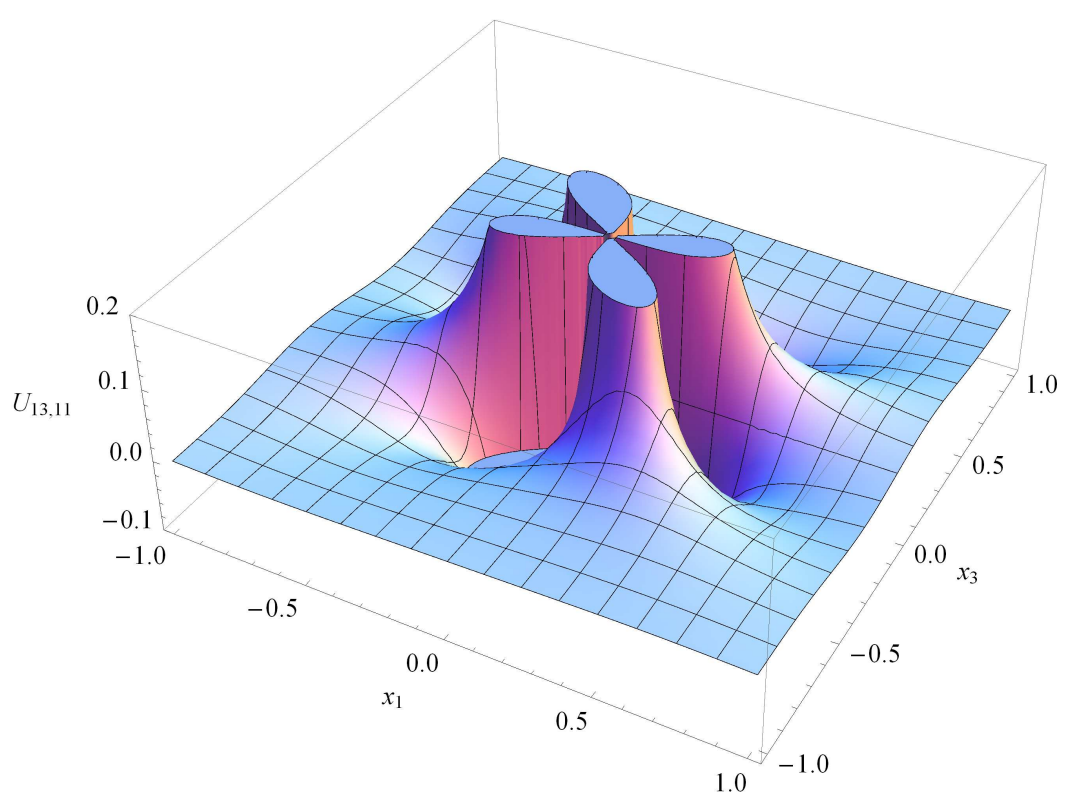

Figure 17: $U_{13,11}$ in plane $x_{2}=0$, for $\triangle<0$. 


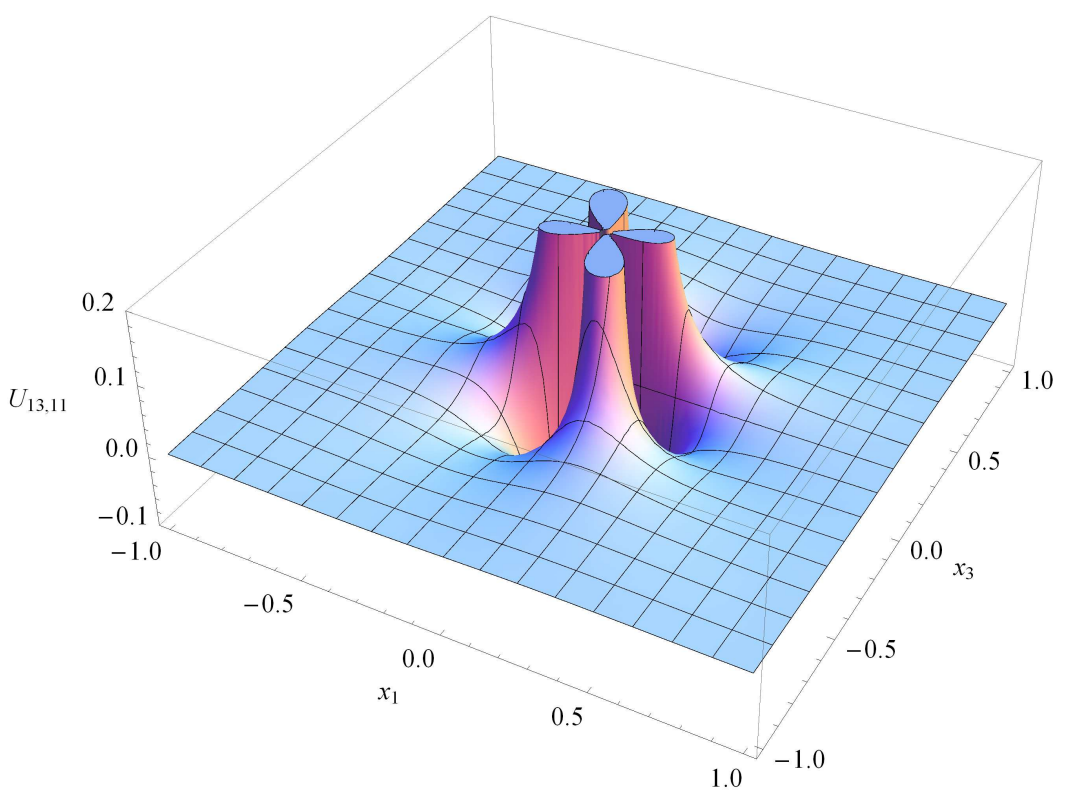

Figure 18: $U_{13,11}$ in plane $x_{2}=0$, for $\triangle=0$.

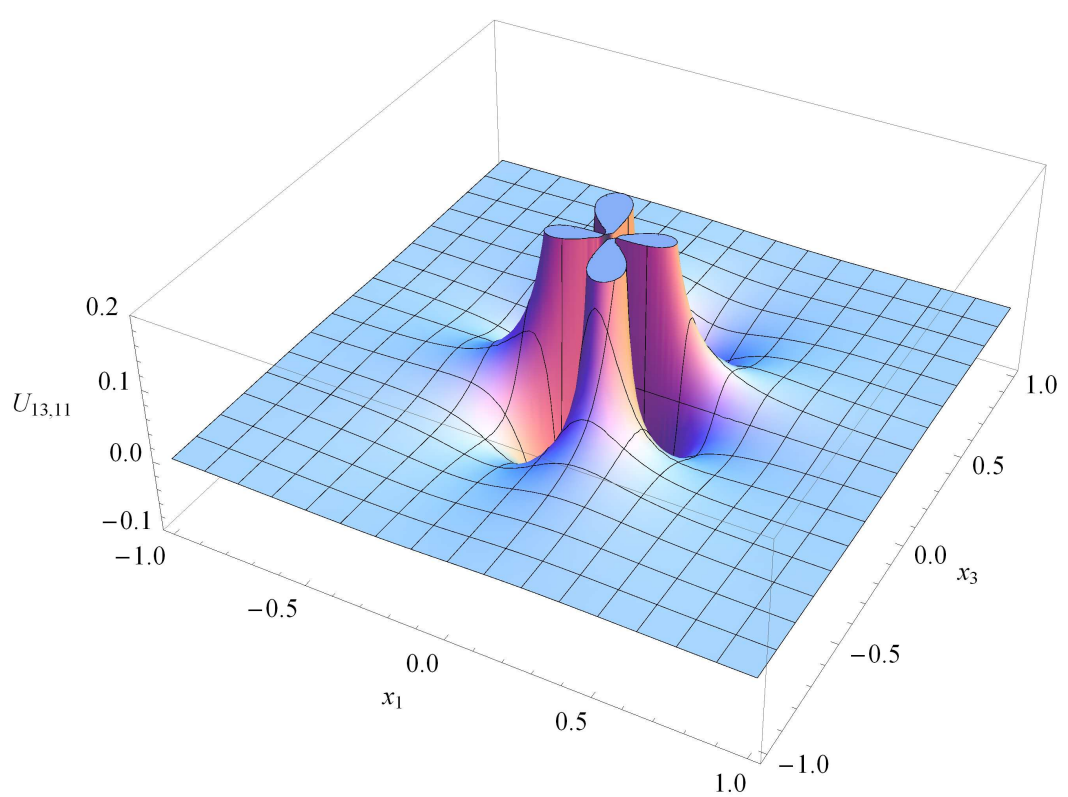

Figure 19: $U_{13,11}$ in plane $x_{2}=0$, for $\triangle>0$. 


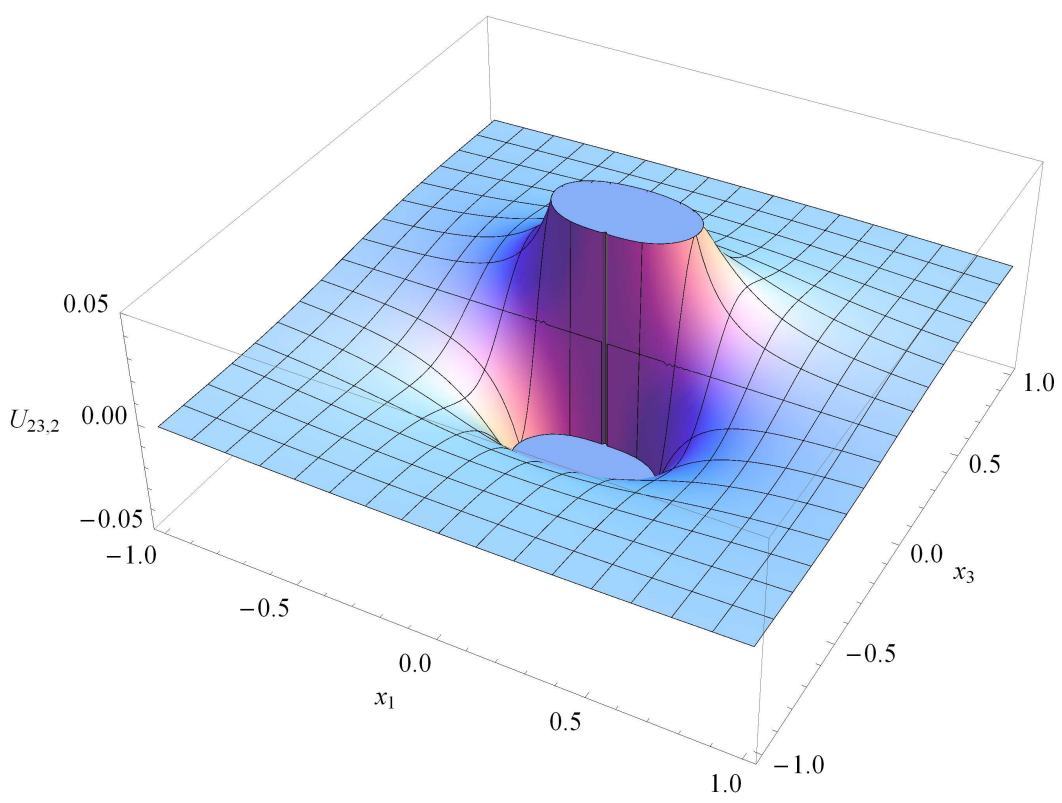

Figure 20: $U_{23,2}$ in plane $x_{2}=0$, for $\triangle<0$.

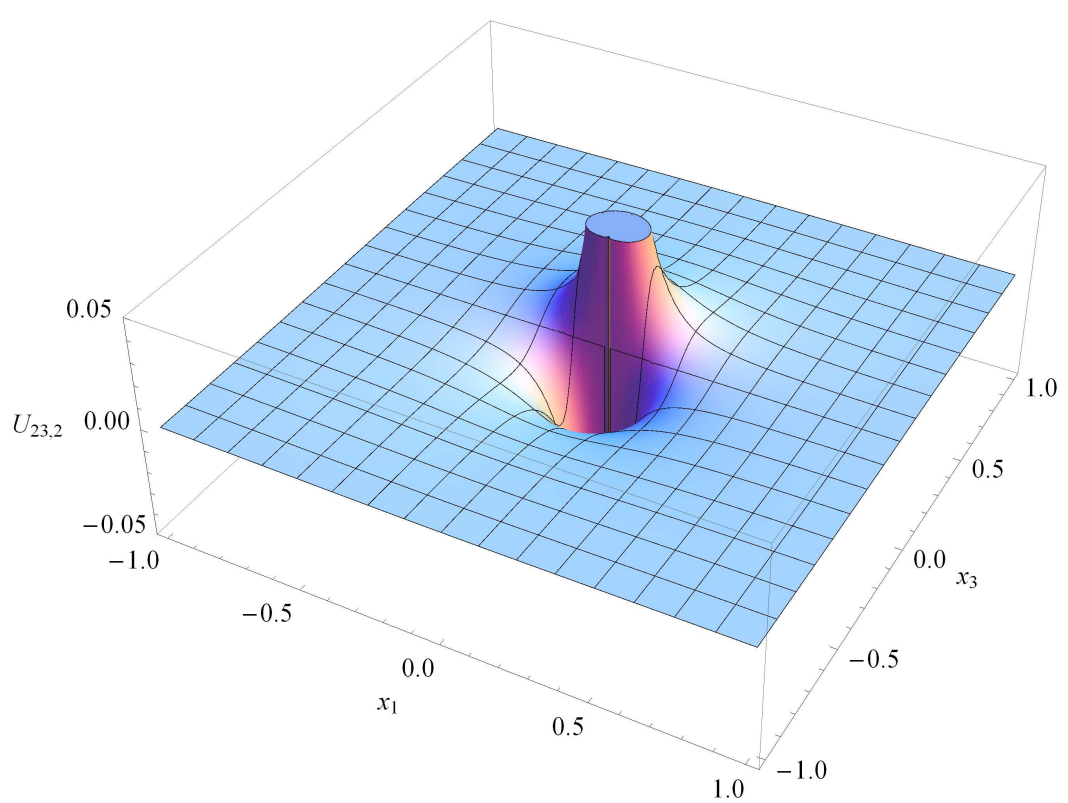

Figure 21: $U_{23,2}$ in plane $x_{2}=0$, for $\triangle=0$. 


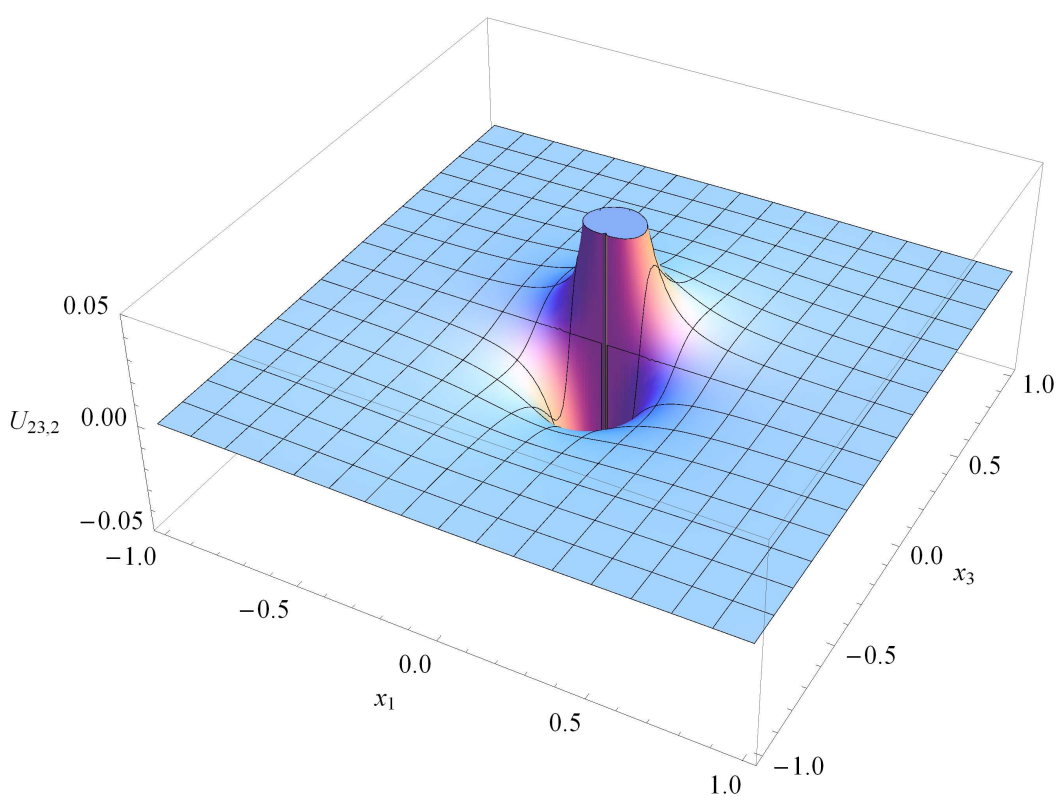

Figure 22: $U_{23,2}$ in plane $x_{2}=0$, for $\triangle>0$.

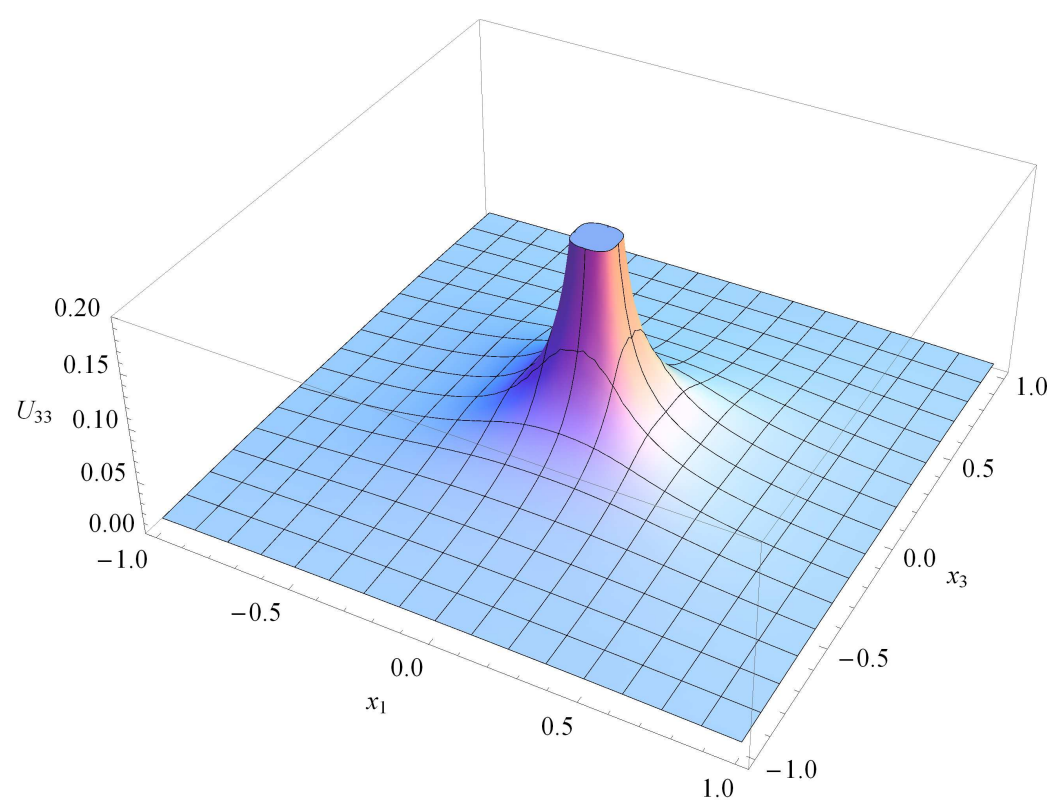

Figure 23: $U_{33}$ in plane $x_{2}=0$, for $\triangle<0$. 


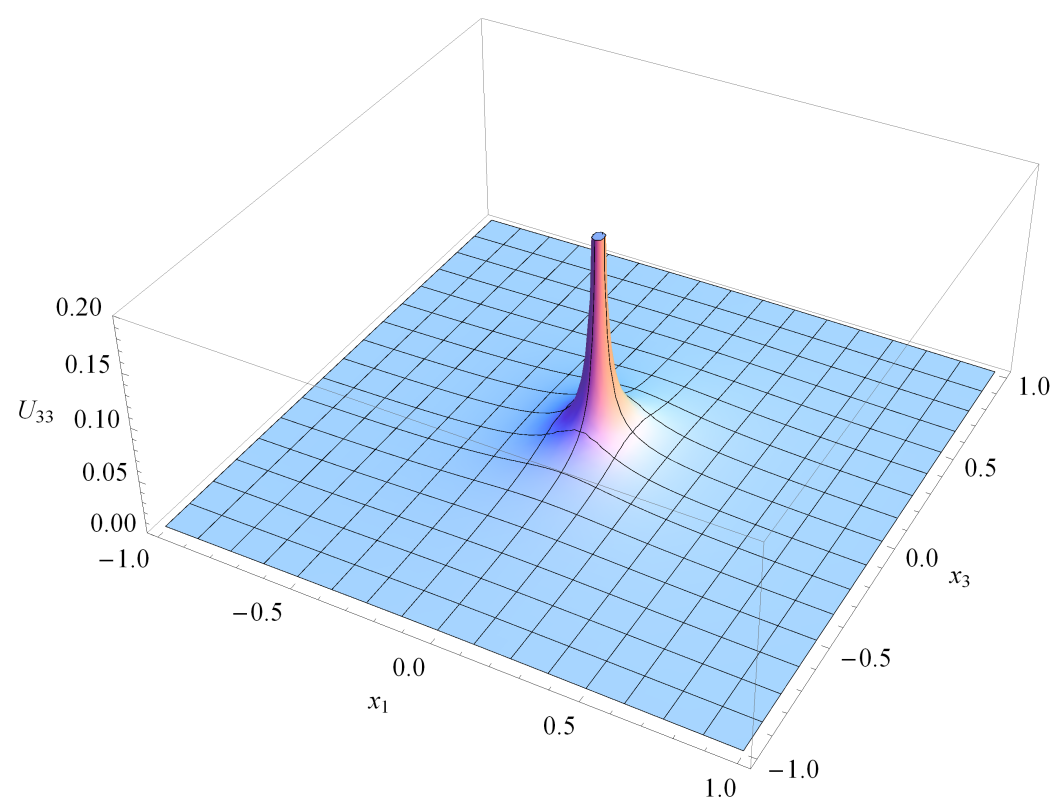

Figure 24: $U_{33}$ in plane $x_{2}=0$, for $\triangle=0$.

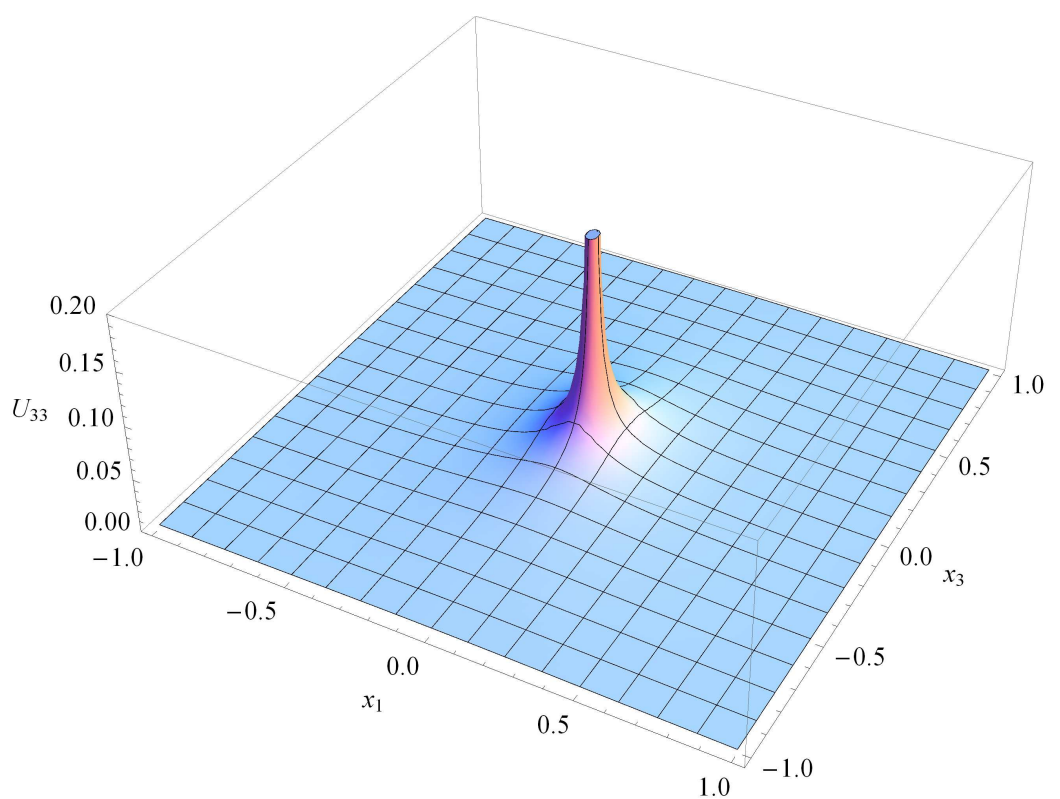

Figure 25: $U_{33}$ in plane $x_{2}=0$, for $\triangle>0$. 


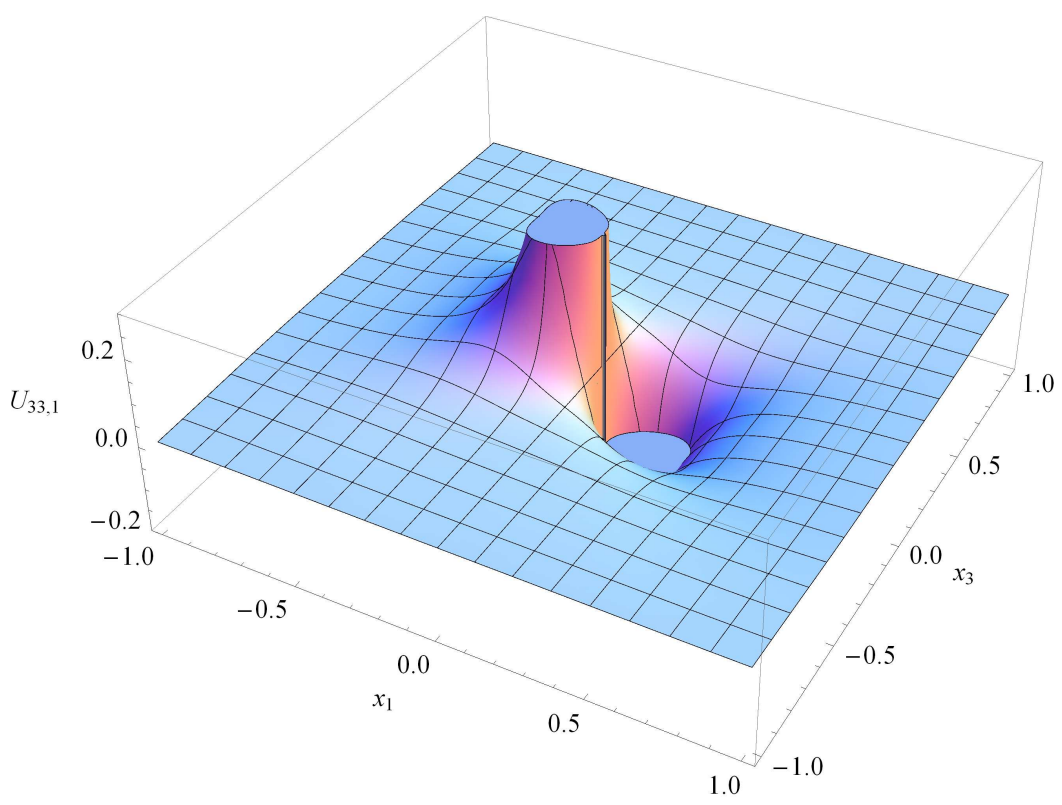

Figure 26: $U_{33,1}$ in plane $x_{2}=0$, for $\triangle<0$.

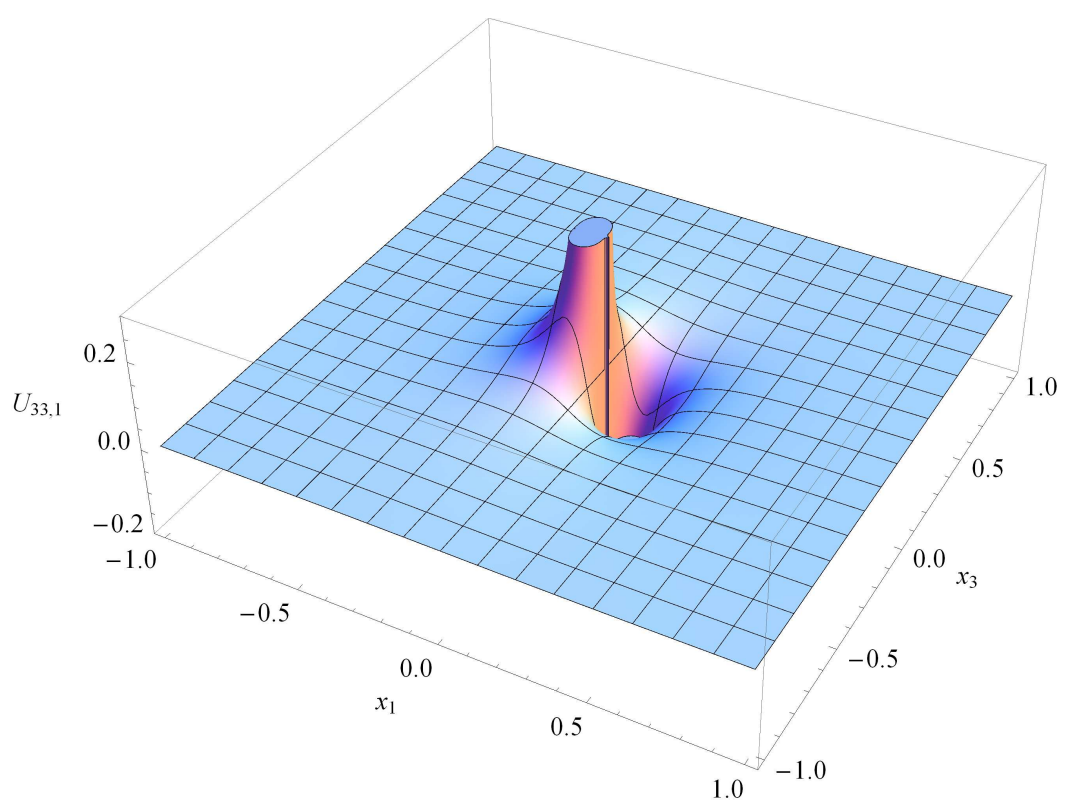

Figure 27: $U_{33,1}$ in plane $x_{2}=0$, for $\triangle=0$. 


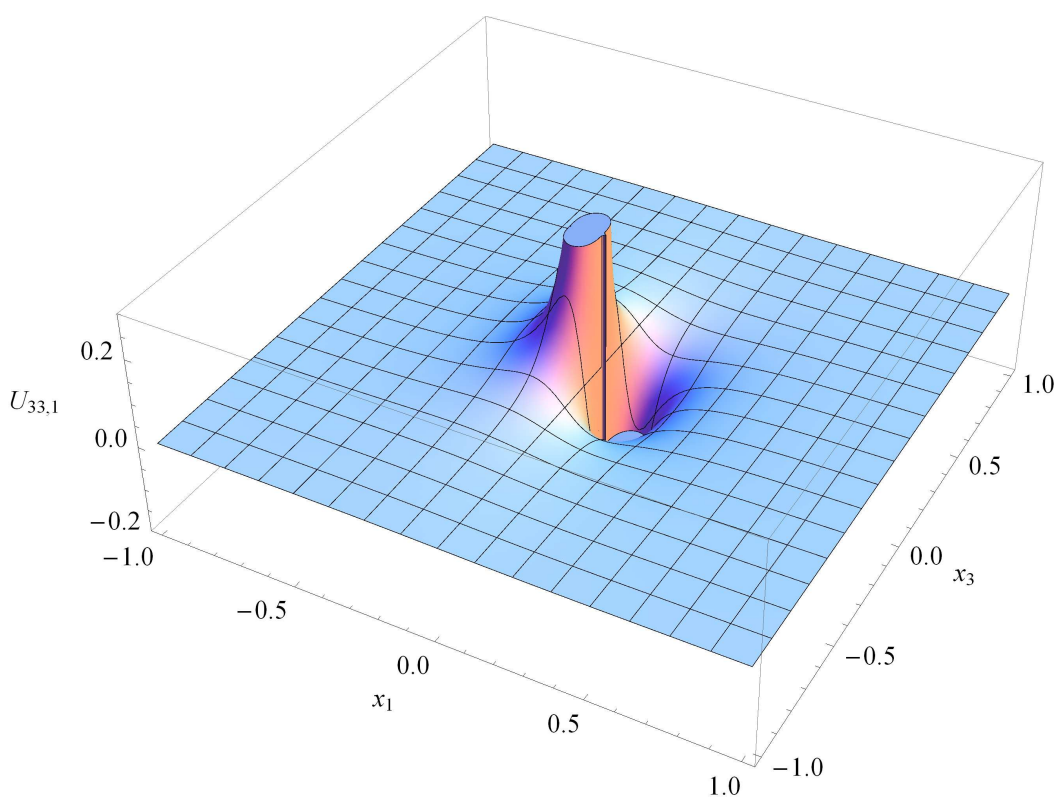

Figure 28: $U_{33,1}$ in plane $x_{2}=0$, for $\triangle>0$.

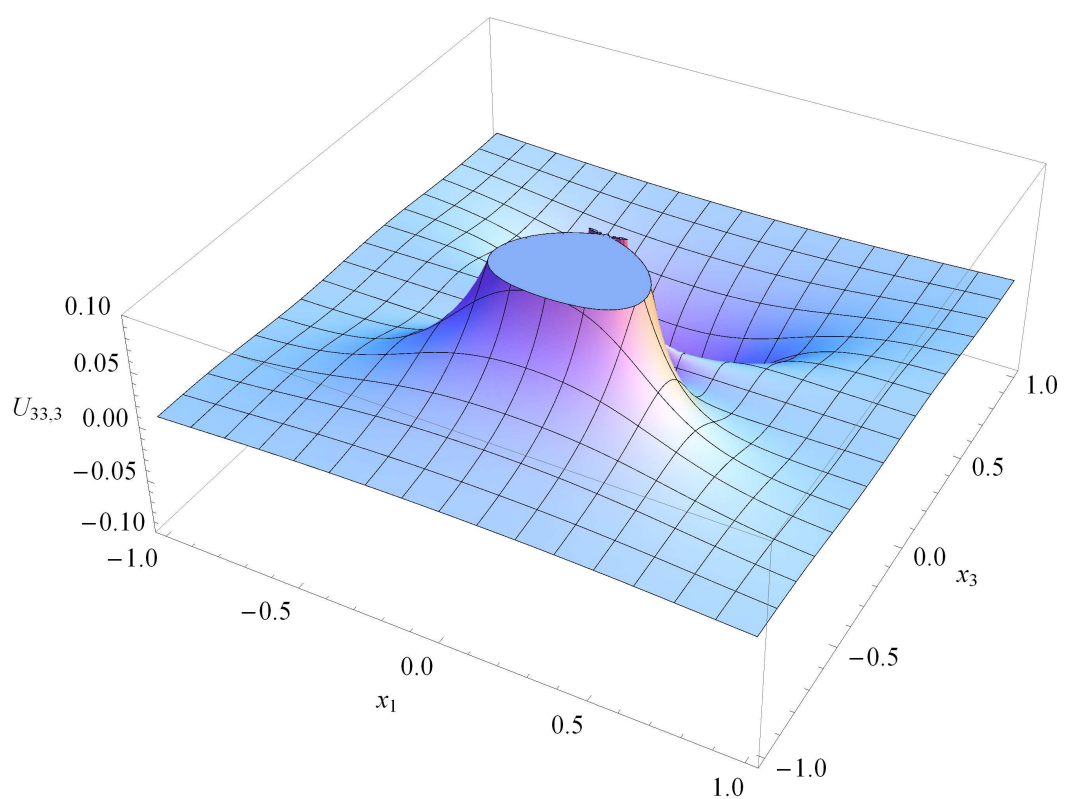

Figure 29: $U_{33,3}$ in plane $x_{2}=0$, for $\triangle<0$. 


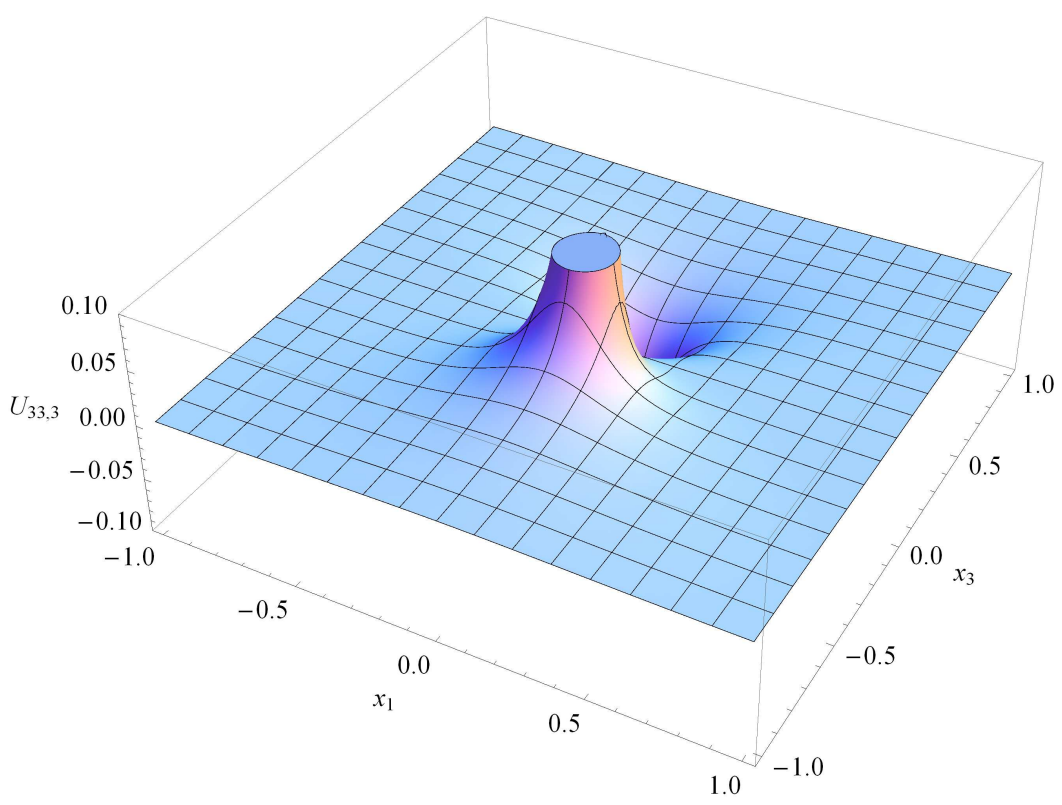

Figure 30: $U_{33,3}$ in plane $x_{2}=0$, for $\triangle=0$.

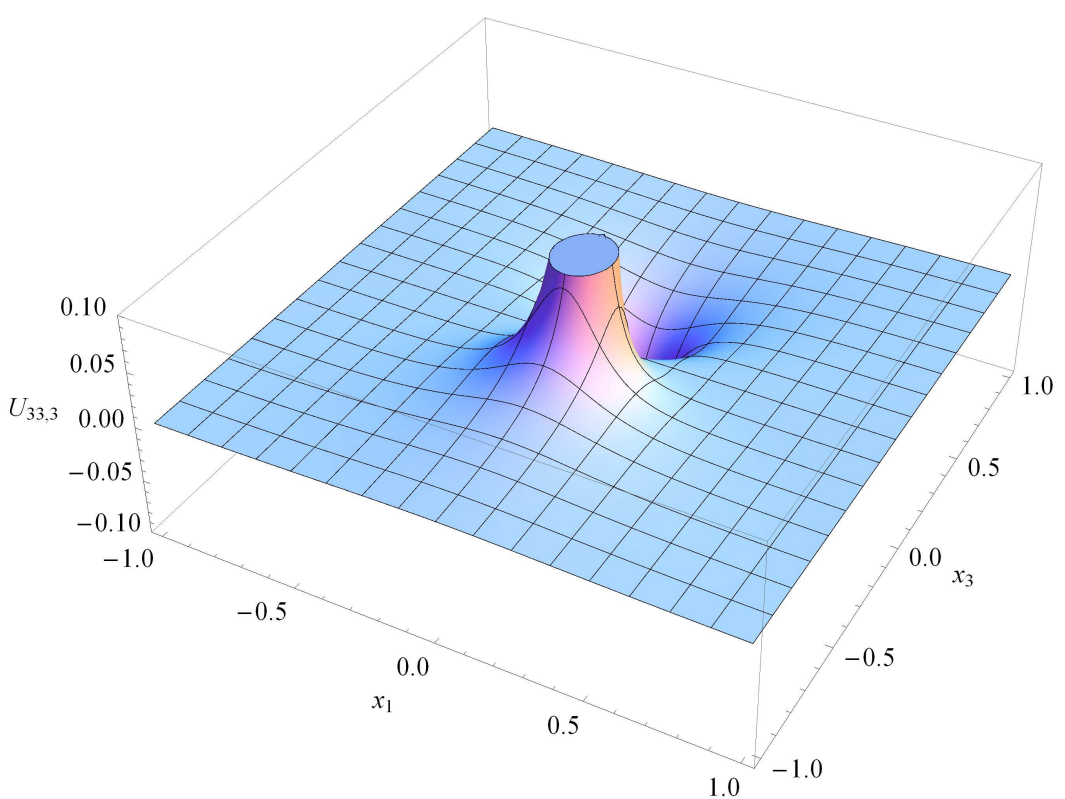

Figure 31: $U_{33,3}$ in plane $x_{2}=0$, for $\triangle>0$. 


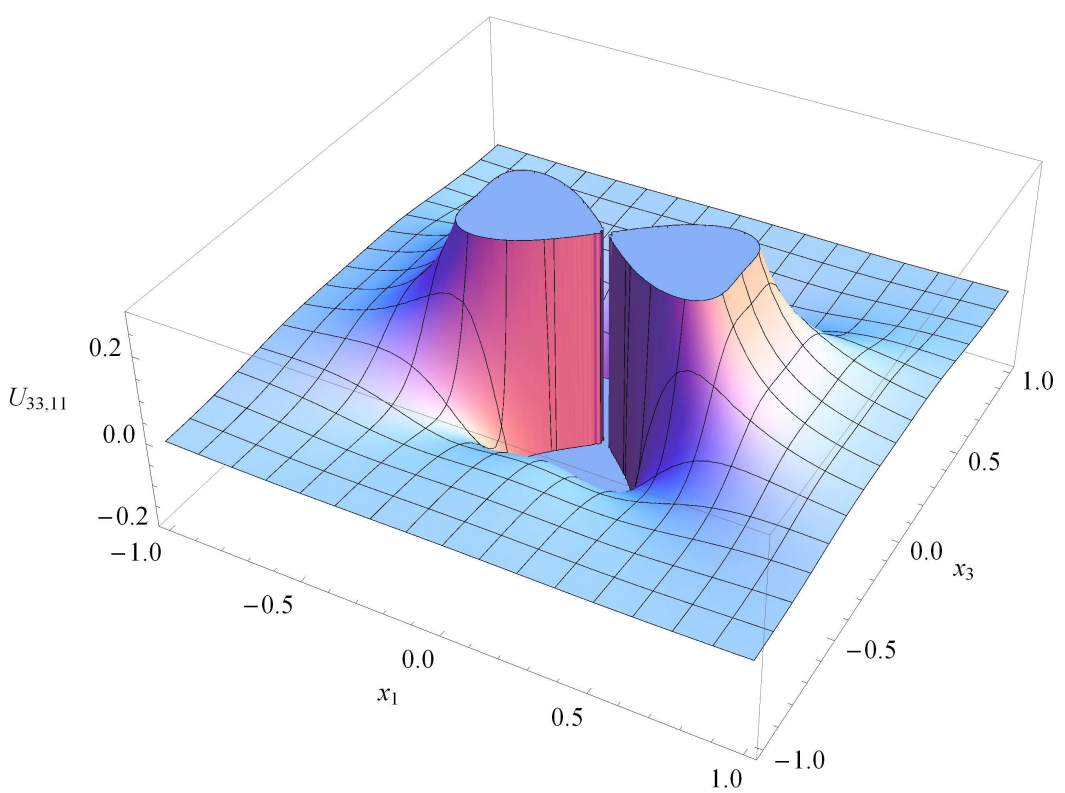

Figure 32: $U_{33,11}$ in plane $x_{2}=0$, for $\triangle<0$.

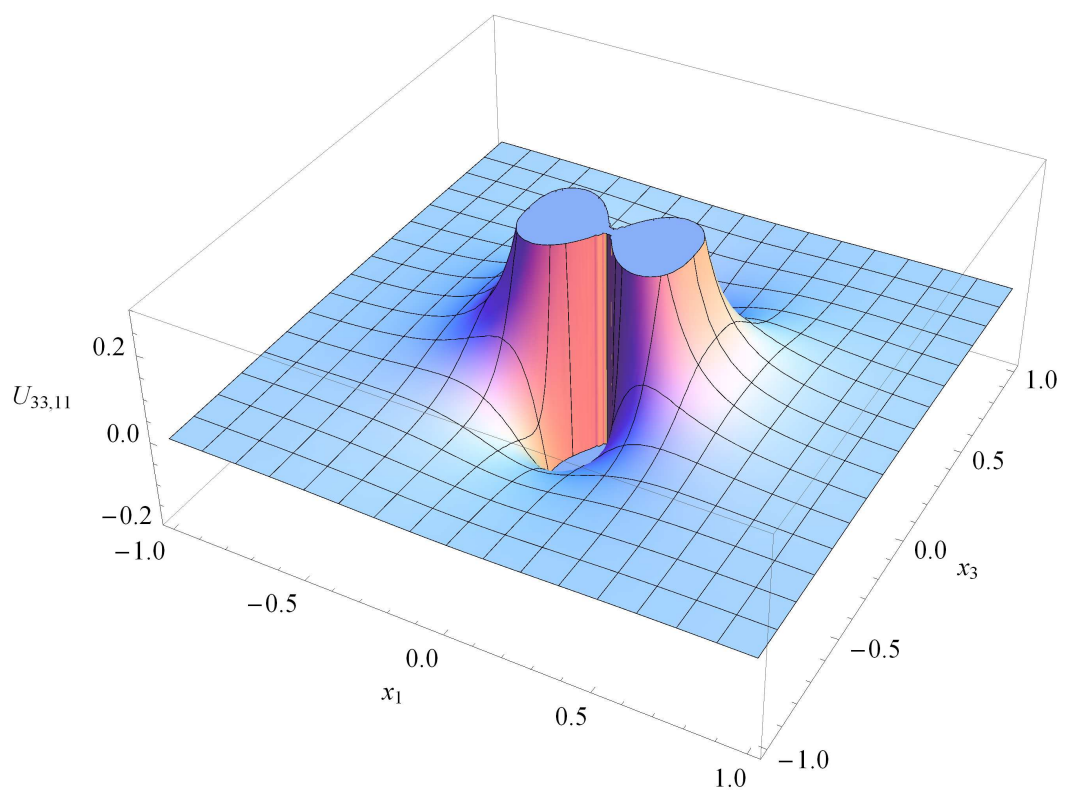

Figure 33: $U_{33,11}$ in plane $x_{2}=0$, for $\triangle=0$. 


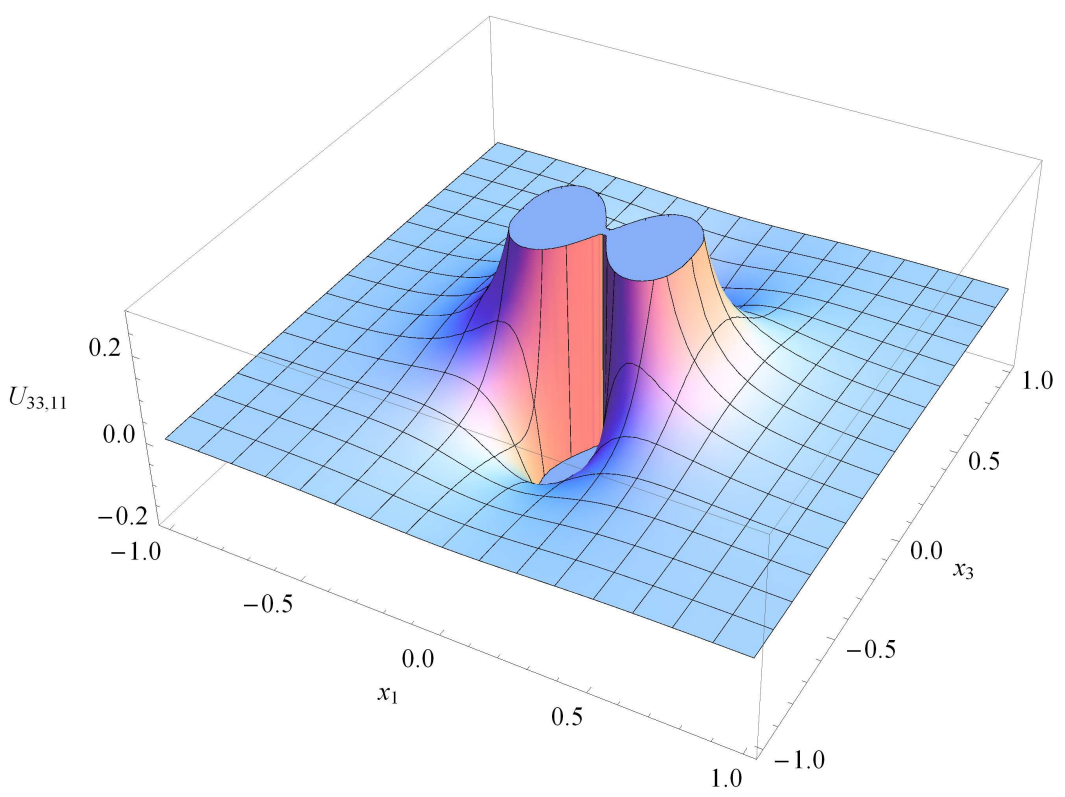

Figure 34: $U_{33,11}$ in plane $x_{2}=0$, for $\triangle>0$.

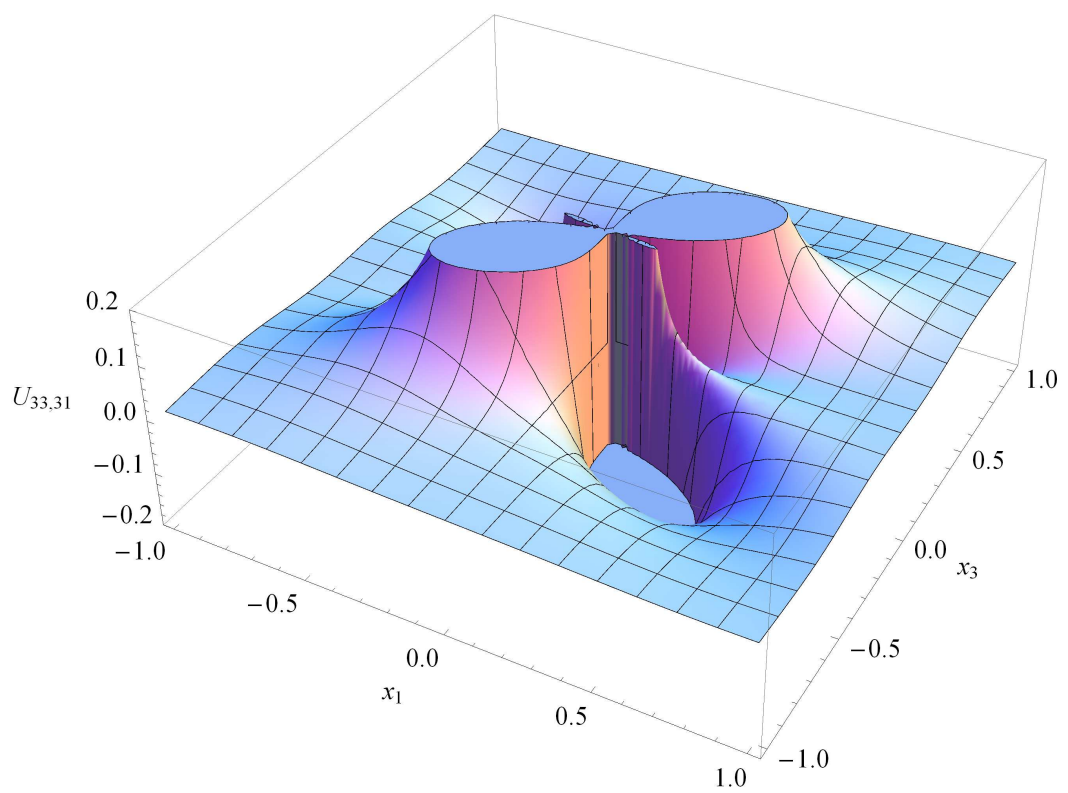

Figure 35: $U_{33,31}=U_{33,13}$ in plane $x_{2}=0$, for $\triangle<0$. 


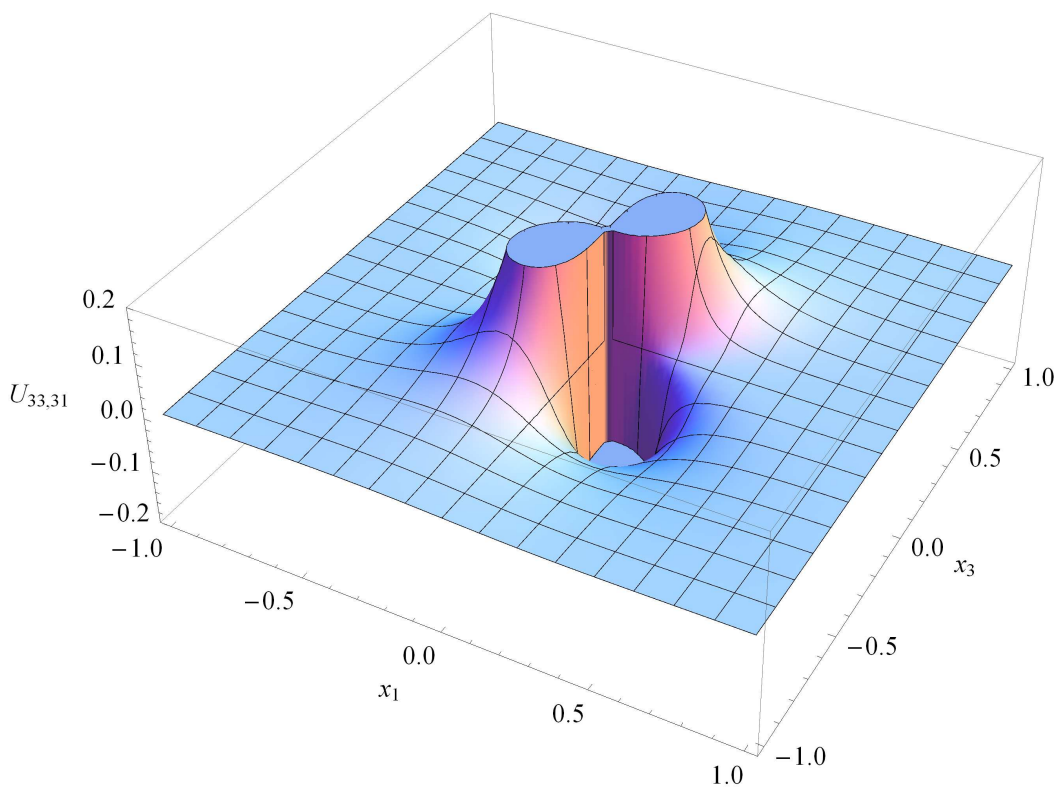

Figure 36: $U_{33,31}=U_{33,13}$ in plane $x_{2}=0$, for $\triangle=0$.

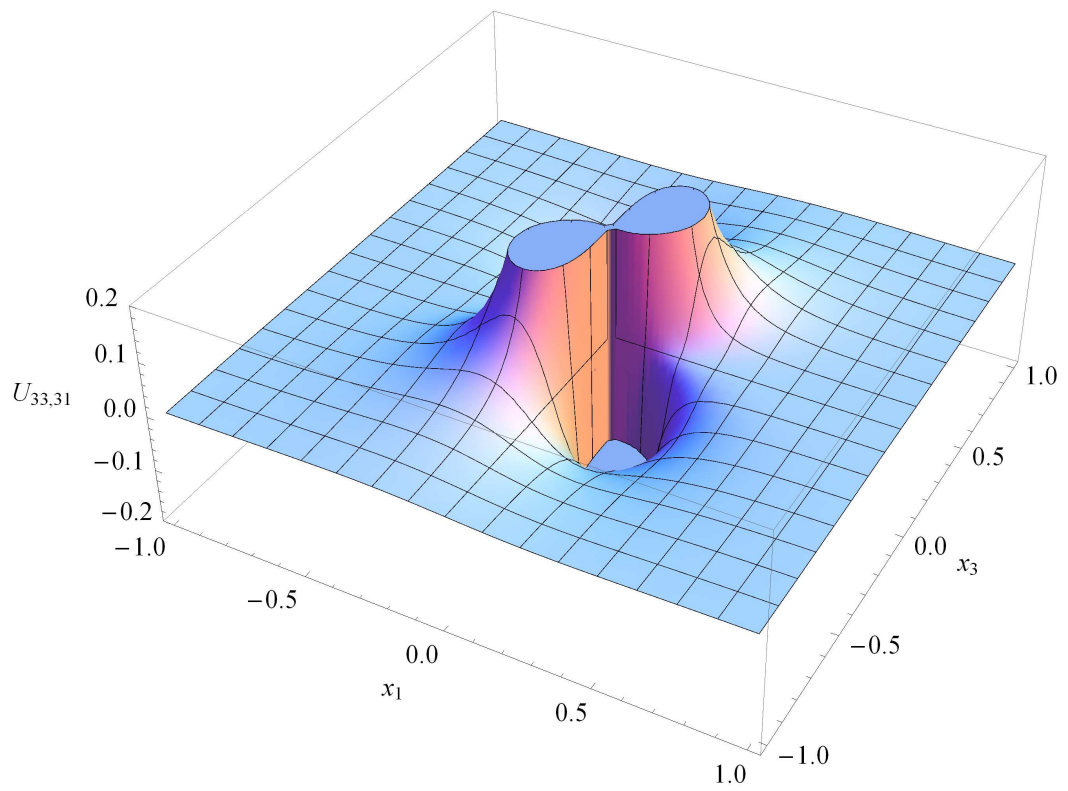

Figure 37: $U_{33,31}=U_{33,13}$ in plane $x_{2}=0$, for $\triangle>0$. 


\title{
APPENDIX B: FORTRAN SUBROUTINE THAT CALCULATES THE FUNDAMENTAL SOLUTION AND ITS DERIVATIVES
}

\author{
Subroutine that calculates the Green Function and its \\ derivatives: $U_{-}\{i k\}, U_{-}\{i k, j\}$ and $U_{-}\{i k, j l\}$ (gu, upf \\ and uppf as defined in the subroutine). The expressions \\ can be used in the displacement and traction boundary \\ integral equations for any $3 D$ transversely isotropic solid.
}

\section{AUTHORS}

L. Tavara, V. Mantic, J.E. Ortiz and F. Paris Group of Elasticity and Strength of Materials Department of Continuum Mechanics

School of Engineering, University of Seville Camino de los Descubrimientos $s / n, 41092$, Seville, Spain

Phone: +34-954487299, 7300

Fax: $\quad+34-954461637$

Corresponding Author:

L. Tavara

ltavara@esi.us.es

http://investigacion.us.es/sisius/sis_showpub.php? idpers $=12449$

Published in:

V. Mantic, L. Tavara, J.E. Ortiz and F. Paris.

Recent developments in the evaluation of $3 D$ fundamental solution and its derivatives for transversely isotropic elastic materials. Electronic Journal of Boundary

Elements. 2012

subroutine UikUikjUikj1(X, c11, c12, c13,c33,c44,c66,gu, upf, uppf)

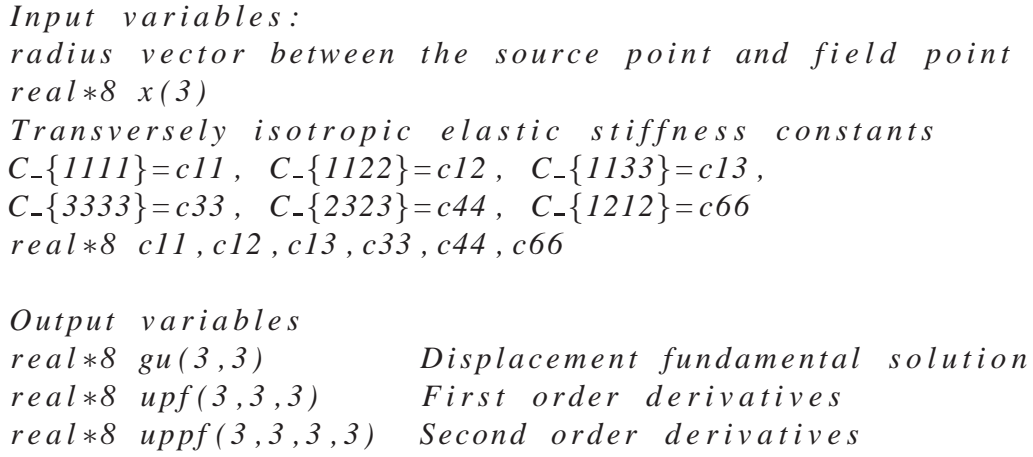


implicit none

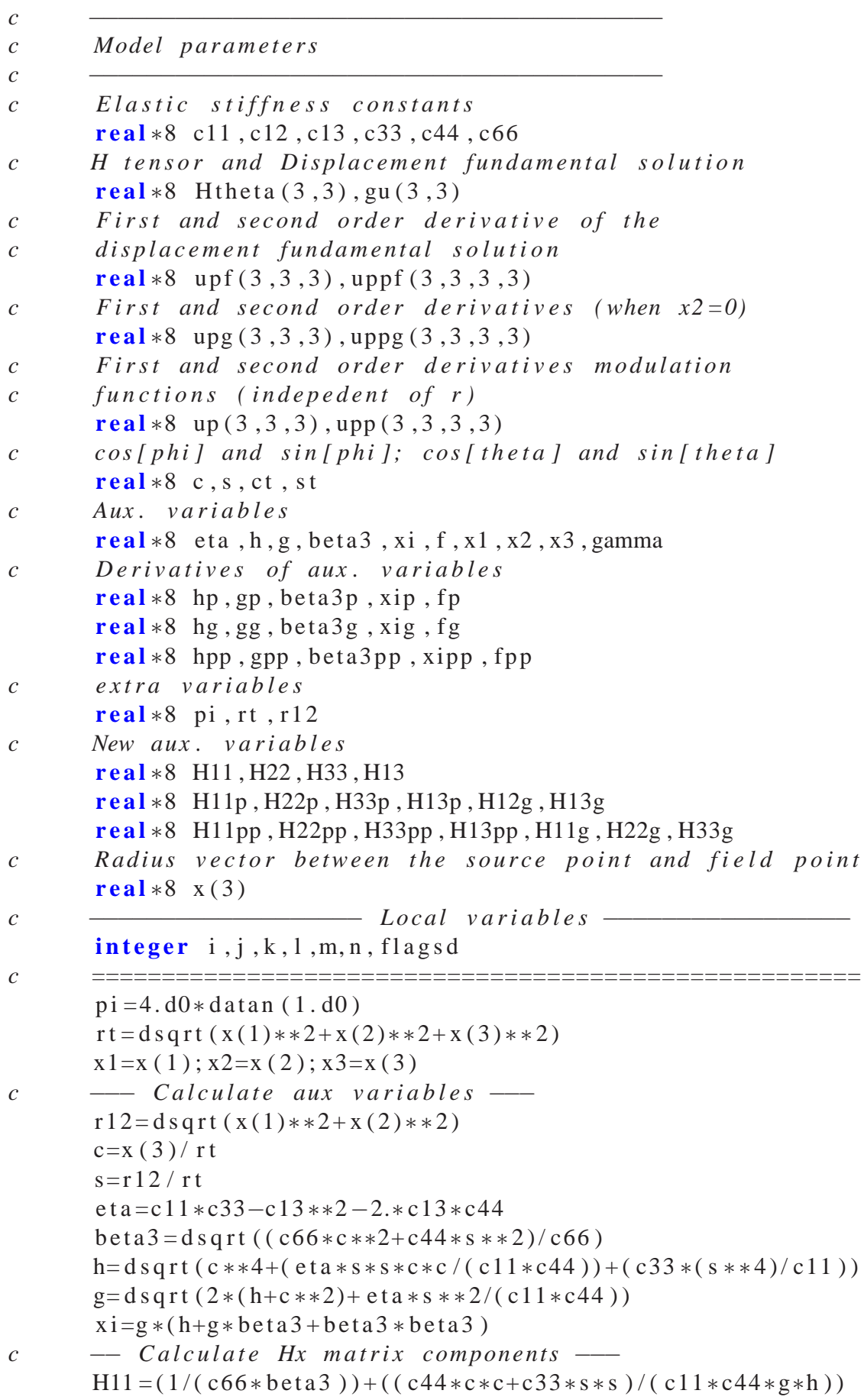




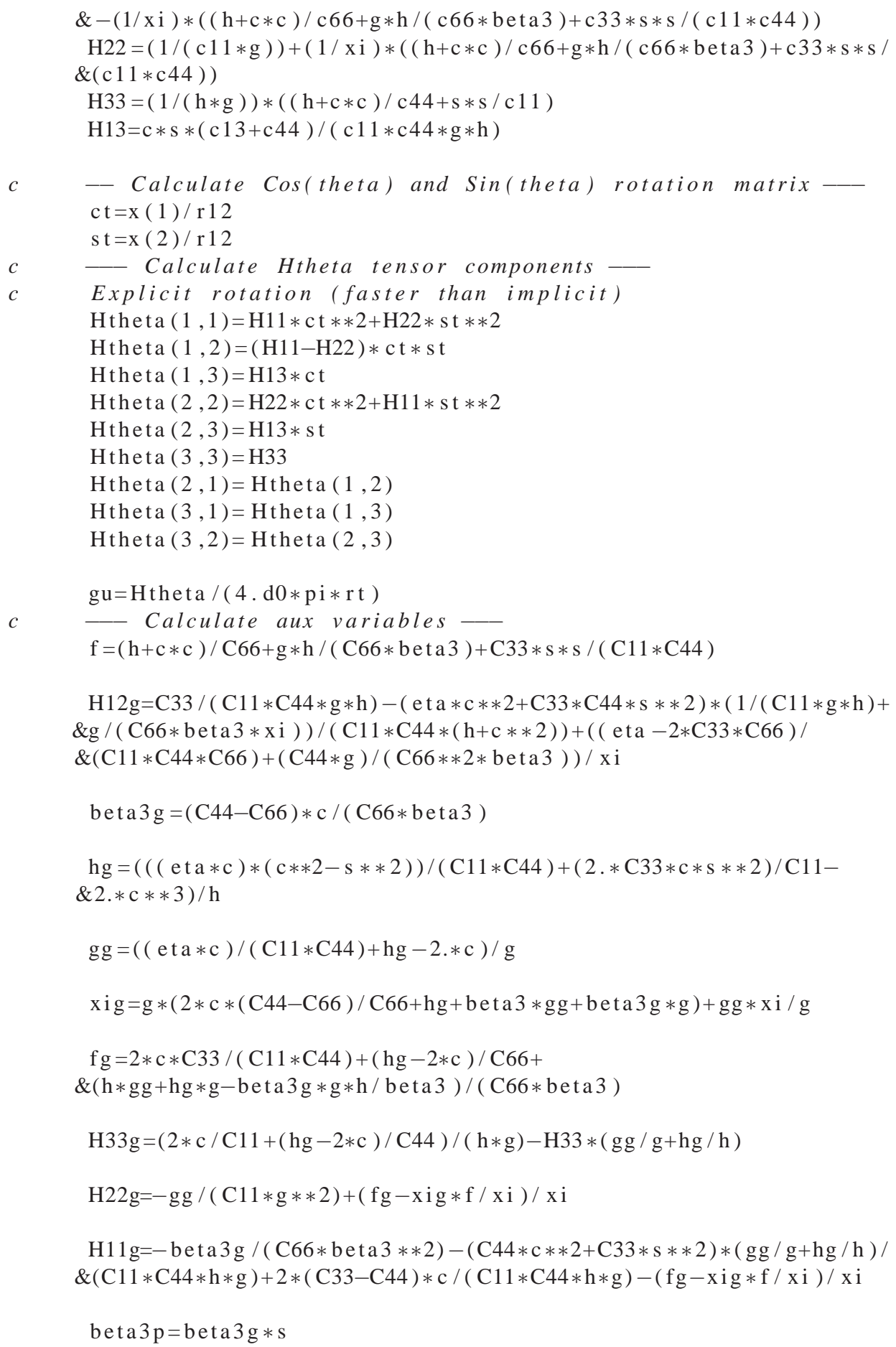




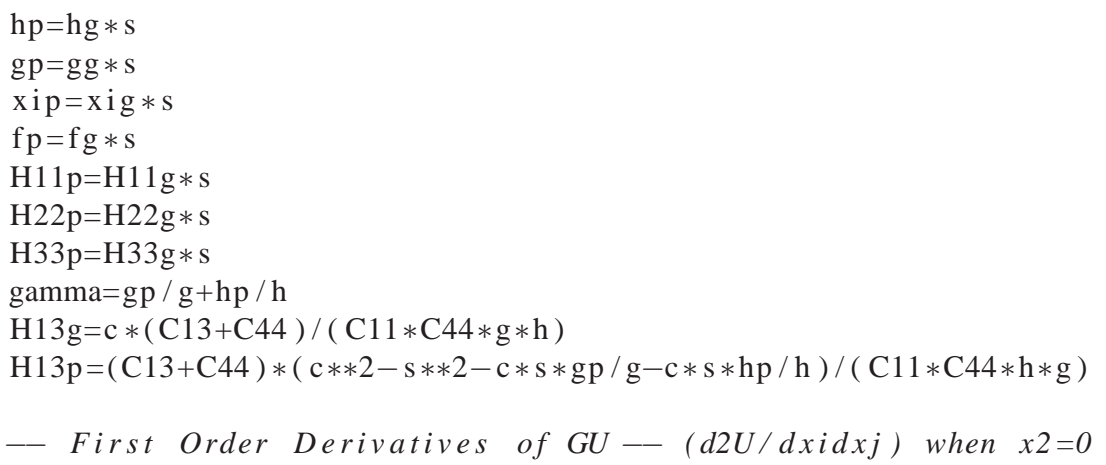


$\operatorname{up}(3,3,2)=\operatorname{upg}(3,3,1) * \mathrm{st}$

up $(3,3,3)=\operatorname{upg}(3,3,3)$

$\operatorname{up}(2,1,1)=\operatorname{up}(1,2,1)$

up $(2,1,2)=\operatorname{up}(1,2,2)$

up $(2,1,3)=\operatorname{up}(1,2,3)$

$\operatorname{up}(3,1,1)=\operatorname{up}(1,3,1)$

up $(3,1,2)=\operatorname{up}(1,3,2)$

up $(3,1,3)=\operatorname{up}(1,3,3)$

up $(3,2,1)=\operatorname{up}(2,3,1)$

up $(3,2,2)=$ up $(2,3,2)$

up $(3,2,3)=\operatorname{up}(2,3,3)$

upf $=$ up / ( $4 . \mathrm{d} 0 * \mathrm{pi} * \mathrm{rt} * * 2)$

Calculate NEW aux variables

beta $3 \mathrm{pp}=-$ beta $3 \mathrm{p} * * 2 /$ beta $3+(\mathrm{C} 44-\mathrm{C} 66) *(\mathrm{c} * * 2-\mathrm{s} * * 2) /(\mathrm{C} 66 *$ beta 3$)$

$\mathrm{hpp}=(-\mathrm{hp} * * 2-2 * \mathrm{~h} * * 2+2 * \mathrm{c} * * 2 * \mathrm{~s} * * 2 *(3+3 * \mathrm{C} 33 / \mathrm{C} 11-\mathrm{eta} /(\mathrm{C} 11 * \mathrm{C} 44))+$ \&eta $*((\mathrm{c} * * 2-\mathrm{s} * * 2) * * 2) /(\mathrm{C} 11 * \mathrm{C} 44)) / \mathrm{h}$

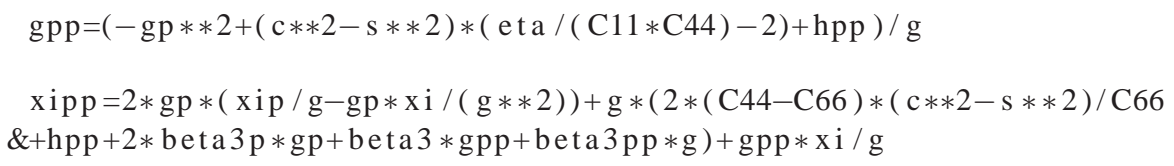

$\mathrm{H} 13 \mathrm{pp}=-\mathrm{H} 13 \mathrm{p} * \mathrm{gamma}+(\mathrm{C} 13+\mathrm{C} 44) *(-4 * \mathrm{c} * \mathrm{~s}-((\mathrm{c} * * 2-\mathrm{s} * * 2) * \mathrm{gp}+\mathrm{c} * \mathrm{~s} * \mathrm{gpp}) / \mathrm{g}-$ $\&((\mathrm{c} * * 2-\mathrm{s} * * 2) * \mathrm{hp}+\mathrm{c} * \mathrm{~s} * \mathrm{hpp}) / \mathrm{h}+\mathrm{c} * \mathrm{~s} *((\mathrm{gp} / \mathrm{g}) * * 2+(\mathrm{hp} / \mathrm{h}) * * 2))$

$\& /(\mathrm{C} 11 * \mathrm{C} 44 * \mathrm{~h} * \mathrm{~g})$

$\mathrm{H} 33 \mathrm{pp}=-(\mathrm{gp} /(\mathrm{h} * \mathrm{~g} * * 2)+\mathrm{hp} /(\mathrm{g} * \mathrm{~h} * * 2)) *(2 * \mathrm{c} * \mathrm{~s} / \mathrm{C} 11+(\mathrm{hp}-2 * \mathrm{c} * \mathrm{~s}) / \mathrm{C} 44)+$ $\&(2 *(\mathrm{c} * * 2-\mathrm{s} * * 2) / \mathrm{C} 11+(\mathrm{hpp}-2 *(\mathrm{c} * * 2-\mathrm{s} * * 2)) / \mathrm{C} 44) /(\mathrm{g} * \mathrm{~h})-\mathrm{H} 33 \mathrm{p} *$ gamma $\& \mathrm{H} 33 *((\mathrm{gpp} * \mathrm{~g}-\mathrm{gp} * * 2) /(\mathrm{g} * * 2)+(\mathrm{hpp} * \mathrm{~h}-\mathrm{hp} * * 2) /(\mathrm{h} * * 2))$

$\mathrm{H} 22 \mathrm{pp}=(2 *(\mathrm{gp} * * 2)-\mathrm{gpp} * \mathrm{~g}) /(\mathrm{C} 11 * \mathrm{~g} * * 3)+\mathrm{xip} *(\mathrm{f} * \mathrm{xip} / \mathrm{xi}-\mathrm{fp}) /(\mathrm{xi} * * 2)+$ $\&(\mathrm{fpp}-((\mathrm{xipp} * \mathrm{f}+\mathrm{xip} * \mathrm{fp}) * \mathrm{xi}-\mathrm{f} * \mathrm{xip} * * 2) /(\mathrm{xi} * * 2)) / \mathrm{xi}$

$\mathrm{H} 11 \mathrm{pp}=(2 *$ beta $3 \mathrm{p} * * 2-$ beta $3 \mathrm{pp} *$ beta 3$) /(\mathrm{C} 66 *$ beta $3 * * 3)-4 * \mathrm{c} * \mathrm{~s} *(\mathrm{C} 33-\mathrm{C} 44)$ $\& *$ gamma $/(\mathrm{C} 11 * \mathrm{C} 44 * \mathrm{~g} * \mathrm{~h})+(\mathrm{C} 44 * \mathrm{c} * * 2+\mathrm{C} 33 * \mathrm{~s} * * 2) *($ gamma $* * 2-$ $\&(\mathrm{gpp} * \mathrm{~g}-\mathrm{gp} * * 2) /(\mathrm{g} * * 2)-(\mathrm{hpp} * \mathrm{~h}-\mathrm{hp} * * 2) /(\mathrm{h} * * 2)) /(\mathrm{C} 11 * \mathrm{C} 44 * \mathrm{~g} * \mathrm{~h})+$ $\& 2 *(\mathrm{C} 33-\mathrm{C} 44) *(\mathrm{c} * * 2-\mathrm{s} * * 2) /(\mathrm{C} 11 * \mathrm{C} 44 * \mathrm{~g} * \mathrm{~h})-\mathrm{xip} *(\mathrm{f} * \mathrm{xip} / \mathrm{xi}-\mathrm{fp}) /(\mathrm{xi} * * 2)-$ $\&(\mathrm{fpp}-((\mathrm{xipp} * \mathrm{f}+\mathrm{xip} * \mathrm{fp}) * \mathrm{xi}-\mathrm{f} * \mathrm{xip} * * 2) /(\mathrm{xi} * * 2)) / \mathrm{xi}$ 


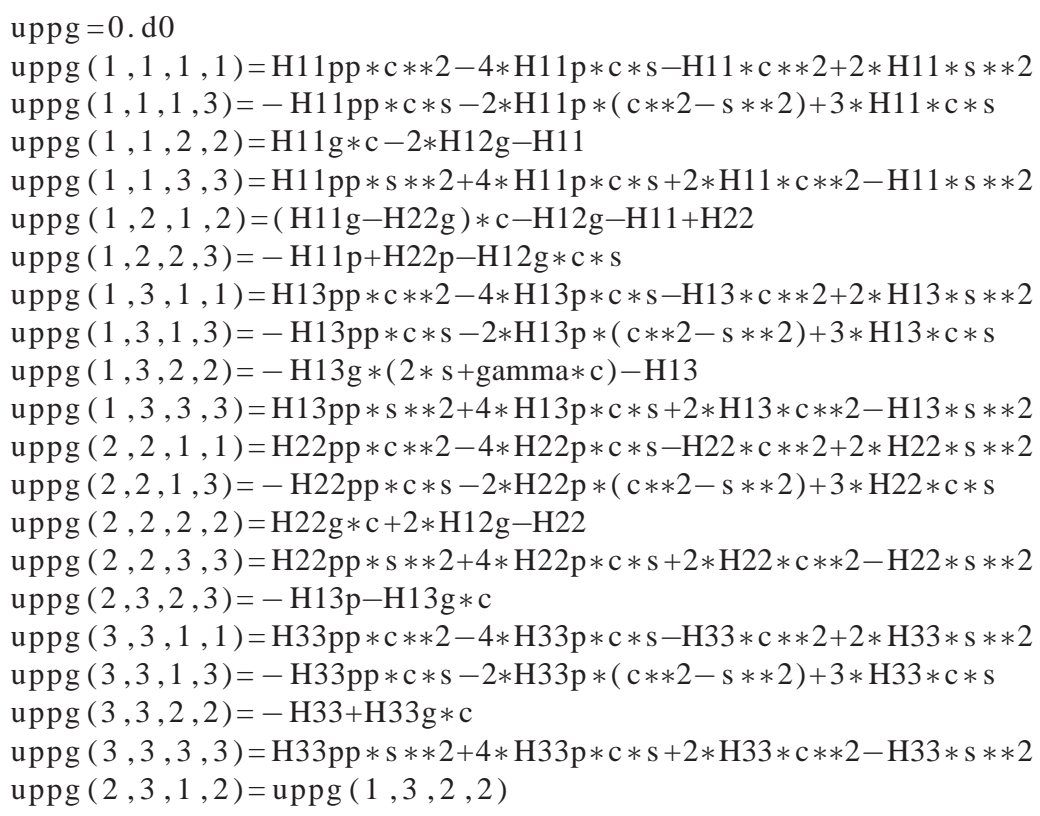

Explicit rotation (faster than implicit)

$\operatorname{upp}(1,1,1,1)=\operatorname{uppg}(1,1,1,1) * \mathrm{ct} * * 4+\operatorname{uppg}(1,1,2,2) * \mathrm{ct} * * 2 * \mathrm{st} * * 2+4$ $\& * \operatorname{uppg}(1,2,1,2) * \mathrm{ct} * * 2 * \mathrm{st} * * 2+\operatorname{uppg}(2,2,1,1) * \mathrm{ct} * * 2 * \mathrm{st} * * 2+\operatorname{uppg}(2,2,2,2)$ $\& * \mathrm{st} * * 4$

$\operatorname{upp}(1,1,1,2)=\operatorname{uppg}(1,1,1,1) * \mathrm{st} * \mathrm{ct} * * 3-\operatorname{uppg}(1,1,2,2) * \mathrm{st} * \mathrm{ct} * * 3$ $\&-2 * \operatorname{uppg}(1,2,1,2) * \mathrm{st} * \mathrm{ct} * * 3+2 * \operatorname{uppg}(1,2,1,2) * \mathrm{ct} * \mathrm{st} * * 3+\mathrm{uppg}(2,2,1,1)$ $\& * \mathrm{ct} * \mathrm{st} * * 3-\operatorname{uppg}(2,2,2,2) * \mathrm{ct} * \mathrm{st} * * 3$

$\operatorname{upp}(1,1,1,3)=\operatorname{uppg}(1,1,1,3) * \mathrm{ct} * * 3+2 * \operatorname{uppg}(1,2,2,3) * \mathrm{ct} * \mathrm{st} * * 2$ \&+uppg $(2,2,1,3) * \mathrm{ct} * \mathrm{st} * * 2$

$\operatorname{upp}(1,1,2,2)=\operatorname{uppg}(1,1,1,1) * \mathrm{ct} * * 2 * \mathrm{st} * * 2+\operatorname{uppg}(1,1,2,2) * \mathrm{ct} * * 4$ $\&-4 * \operatorname{uppg}(1,2,1,2) * \mathrm{ct} * * 2 * \mathrm{st} * * 2+\operatorname{uppg}(2,2,1,1) * \mathrm{st} * * 4+\operatorname{uppg}(2,2,2,2)$ $\& * \mathrm{ct} * * 2 * \mathrm{st} * * 2$

$\operatorname{upp}(1,1,2,3)=\operatorname{uppg}(1,1,1,3) * \mathrm{st} * \mathrm{ct} * * 2-2 * \operatorname{uppg}(1,2,2,3) * \mathrm{st} * \mathrm{ct} * * 2$ $\&+\operatorname{uppg}(2,2,1,3) * \mathrm{st} * * 3$

$\operatorname{upp}(1,1,3,3)=\operatorname{uppg}(1,1,3,3) * \mathrm{ct} * * 2+\operatorname{uppg}(2,2,3,3) * \mathrm{st} * * 2$

$\operatorname{upp}(1,2,1,1)=\operatorname{uppg}(1,1,1,1) * \mathrm{st} * \mathrm{ct} * * 3+\operatorname{uppg}(1,1,2,2) * \mathrm{ct} * \mathrm{st} * * 3$ $\&-2 * \operatorname{uppg}(1,2,1,2) * \mathrm{st} * \mathrm{ct} * * 3+2 * \operatorname{uppg}(1,2,1,2) * \mathrm{ct} * \mathrm{st} * * 3-\operatorname{uppg}(2,2,1,1)$ $\& * \mathrm{st} * \mathrm{ct} * * 3-\operatorname{uppg}(2,2,2,2) * \mathrm{ct} * \mathrm{st} * * 3$

$\operatorname{upp}(1,2,1,2)=\operatorname{uppg}(1,1,1,1) * \mathrm{ct} * * 2 * \mathrm{st} * * 2-\operatorname{uppg}(1,1,2,2) * \mathrm{ct} * * 2 * \mathrm{st} * * 2$ 


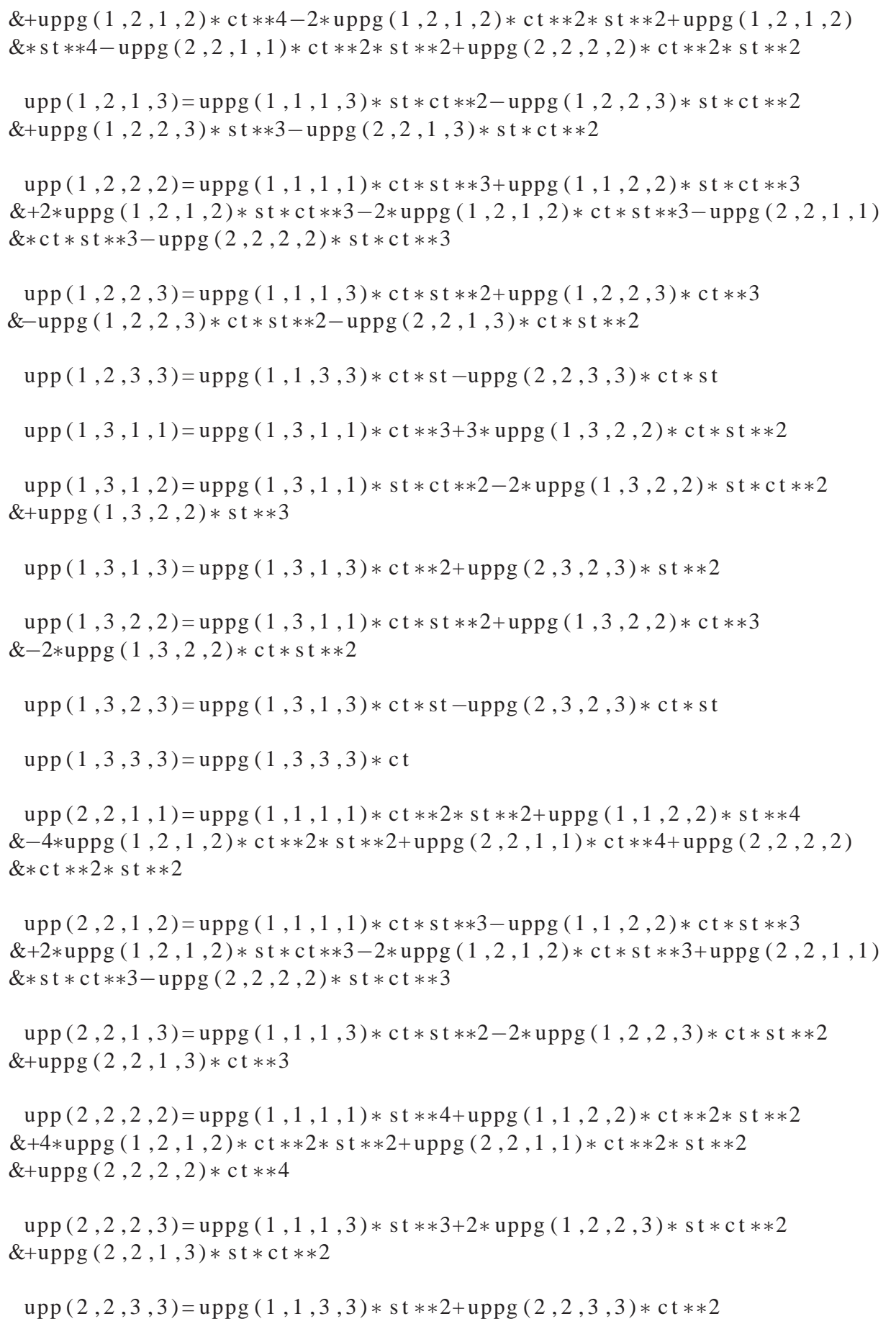


$\operatorname{upp}(2,3,1,1)=\operatorname{uppg}(1,3,1,1) * \mathrm{st} * \mathrm{ct} * * 2-2 * \operatorname{uppg}(1,3,2,2) * \mathrm{st} * \mathrm{ct} * * 2$ $\&+u p p g(1,3,2,2) * \mathrm{st} * * 3$

$\operatorname{upp}(2,3,1,2)=\operatorname{uppg}(1,3,1,1) * \mathrm{ct} * \mathrm{st} * * 2+\operatorname{uppg}(1,3,2,2) * \mathrm{ct} * * 3$ $\&-2 * \operatorname{uppg}(1,3,2,2) * \mathrm{ct} * \mathrm{st} * * 2$

$\operatorname{upp}(2,3,1,3)=\operatorname{uppg}(1,3,1,3) * \mathrm{ct} * \mathrm{st}-\operatorname{uppg}(2,3,2,3) * \mathrm{ct} * \mathrm{st}$

$\operatorname{upp}(2,3,2,2)=\operatorname{uppg}(1,3,1,1) * \mathrm{st} * * 3+3 * \operatorname{uppg}(1,3,2,2) * \mathrm{st} * \mathrm{ct} * * 2$

$\operatorname{upp}(2,3,2,3)=\operatorname{uppg}(1,3,1,3) * \mathrm{st} * * 2+\operatorname{uppg}(2,3,2,3) * \operatorname{ct} * * 2$

$\operatorname{upp}(2,3,3,3)=\operatorname{uppg}(1,3,3,3) * \mathrm{st}$

$\operatorname{upp}(3,3,1,1)=\operatorname{uppg}(3,3,1,1) * \mathrm{ct} * * 2+\operatorname{uppg}(3,3,2,2) * \mathrm{st} * * 2$

$\operatorname{upp}(3,3,1,2)=\operatorname{uppg}(3,3,1,1) * \mathrm{ct} * \mathrm{st}-\operatorname{uppg}(3,3,2,2) * \mathrm{ct} * \mathrm{st}$

$\operatorname{upp}(3,3,1,3)=\operatorname{uppg}(3,3,1,3) * \mathrm{ct}$

$\operatorname{upp}(3,3,2,2)=\operatorname{uppg}(3,3,1,1) * \mathrm{st} * * 2+\operatorname{uppg}(3,3,2,2) * \mathrm{ct} * * 2$

$\operatorname{upp}(3,3,2,3)=\operatorname{uppg}(3,3,1,3) * \mathrm{st}$

$\operatorname{upp}(3,3,3,3)=\operatorname{uppg}(3,3,3,3)$

\section{Symmetry}

$\operatorname{upp}(1,1,2,1)=\operatorname{upp}(1,1,1,2) ; \operatorname{upp}(1,1,3,1)=\operatorname{upp}(1,1,1,3)$ $\operatorname{upp}(1,1,3,2)=\operatorname{upp}(1,1,2,3) ; \operatorname{upp}(1,2,2,1)=\operatorname{upp}(1,2,1,2)$ $\operatorname{upp}(1,2,3,1)=\operatorname{upp}(1,2,1,3) ; \operatorname{upp}(1,2,3,2)=\operatorname{upp}(1,2,2,3)$ $\operatorname{upp}(1,3,2,1)=\operatorname{upp}(1,3,1,2) ; \operatorname{upp}(1,3,3,1)=\operatorname{upp}(1,3,1,3)$ $\operatorname{upp}(1,3,3,2)=\operatorname{upp}(1,3,2,3) ; \operatorname{upp}(2,2,2,1)=\operatorname{upp}(2,2,1,2)$ $\operatorname{upp}(2,2,3,1)=\operatorname{upp}(2,2,1,3) ; \operatorname{upp}(2,2,3,2)=\operatorname{upp}(2,2,2,3)$ $\operatorname{upp}(2,3,2,1)=\operatorname{upp}(2,3,1,2) ; \operatorname{upp}(2,3,3,1)=\operatorname{upp}(2,3,1,3)$ $\operatorname{upp}(2,3,3,2)=\operatorname{upp}(2,3,2,3) ; \operatorname{upp}(3,3,2,1)=\operatorname{upp}(3,3,1,2)$ $\operatorname{upp}(3,3,3,1)=\operatorname{upp}(3,3,1,3) ; \operatorname{upp}(3,3,3,2)=\operatorname{upp}(3,3,2,3)$

$\operatorname{upp}(3,1,1,1)=\operatorname{upp}(1,3,1,1) ; \operatorname{upp}(3,1,1,2)=\operatorname{upp}(1,3,1,2)$ $\operatorname{upp}(3,1,1,3)=\operatorname{upp}(1,3,1,3) ; \operatorname{upp}(3,1,2,1)=\operatorname{upp}(1,3,2,1)$ $\operatorname{upp}(3,1,2,2)=\operatorname{upp}(1,3,2,2) ; \operatorname{upp}(3,1,2,3)=\operatorname{upp}(1,3,2,3)$ $\operatorname{upp}(3,1,3,1)=\operatorname{upp}(1,3,3,1) ; \operatorname{upp}(3,1,3,2)=\operatorname{upp}(1,3,3,2)$ $\operatorname{upp}(3,1,3,3)=\operatorname{upp}(1,3,3,3)$

$\operatorname{upp}(3,2,1,1)=\operatorname{upp}(2,3,1,1) ; \operatorname{upp}(3,2,1,2)=\operatorname{upp}(2,3,1,2)$ upp $(3,2,1,3)=\operatorname{upp}(2,3,1,3) ; \operatorname{upp}(3,2,2,1)=\operatorname{upp}(2,3,2,1)$ $\operatorname{upp}(3,2,2,2)=\operatorname{upp}(2,3,2,2) ; \operatorname{upp}(3,2,2,3)=\operatorname{upp}(2,3,2,3)$ $\operatorname{upp}(3,2,3,1)=\operatorname{upp}(2,3,3,1) ; \operatorname{upp}(3,2,3,2)=\operatorname{upp}(2,3,3,2)$ $\operatorname{upp}(3,2,3,3)=\operatorname{upp}(2,3,3,3)$ 


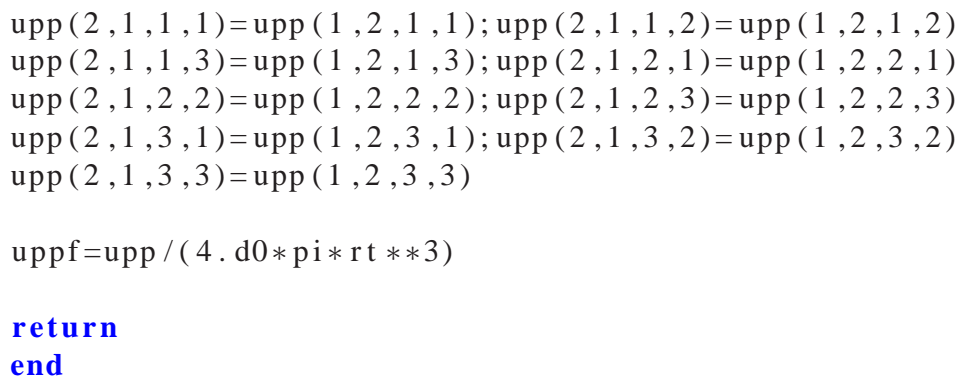

\section{Acknowledgments}

This work was supported by the Consejería de Innovación, Ciencia y Empresa de la Junta de Andalucía, Projects of Excellence TEP-1207, TEP-2045 and TEP-4051, and by the Ministerio de Ciencia e Innovación, Spain, Research Projects TRA2006-08077 and MAT2009-14022.

\section{References}

[1] Távara L, Ortiz J E, Mantič V, París F. Unique real-variable expressions of displacement and traction fundamental solutions covering all transversely isotropic materials for 3D BEM. International Journal for Numerical Methods in Engineering; 74:776798, 2008.

[2] Távara L, Mantič V, Ortiz J E, París F. Unique real-variable expressions of the integral kernels in the Somigliana stress identity covering all transversely isotropic elastic materials for 3D BEM. Computer Methods in Applied Mechanics and Engineering (submitted).

[3] Fredholm I. Sur les équations de l'équilibre d'um corps solide élastique. Acta Mathematica; 23:1-42, 1900.

[4] Lifshitz IM, Rozentsweig LN. Construction of the Green tensor for the fundamental equation of elasticity theory in the case of unbounded elastically anisotropic medium. Zhurnal Eksper. i Teoreticheskoi Fiziki; 17:783-791, 1947.

[5] Ting TCT. Anisotropic Elasticity Theory and Applications. Oxford University Press: Oxford, 1996.

[6] Malén K. A unified six-dimensional treatment of elastic Green's functions and dislocations. Physica Status Solidi B; 44: 661-672, 1971.

[7] Barnett DM. The precise evaluation of derivatives of the anisotropic elastic Green's functions. Phys. Stat. Sol. (b); 49:741748, 1972. 
[8] Nakamura G, Tanuma K. A formula for the fundamental solution of anisotropic elasticity. Quarterly Journal of Mechanics and Applied Mathematics; 50:179-194, 1997.

[9] Ting TCT, Lee VG. The three-dimensional elastostatic Green's function for general anisotropic linear elastic solids. Quarterly Journal of Mechanics and Applied Mathematics; 50:407-426, 1997.

[10] Lee VG. Explicit expression of derivatives of elastic Green's functions for general anisotropic materials. Mechanics Research Communications; 30: 241-249, 2003.

[11] Lee VG. Derivatives of the three-dimensional Greens functions for anisotropic materials. International Journal of Solids and Structuress; 46: 3471-3479, 2009.

[12] Tanuma K. Stroh Formalism and Rayleigh Waves Journal of Elasticity; 89:1-3, 2007.

[13] Shiah YC, Tan CL, Lee VG. Evaluation of explicit-form fundamental solutions for displacements and stresses in 3D anisotropic elastic solids. Computer Modeling in Engineering and Sciences; 34:205-226, 2008.

[14] Tan CL, Shiah Y C, Lin CW. Stress analysis of 3D generally anisotropic elastic solids using the boundary element method. Computer Modeling in Engineering and Sciences; 41:195-214, 2009.

[15] Shiah YC, Tan CL, Lee VG. Internal point solutions for displacements and stresses in 3D anisotropic elastic solids using the boundary element method. Computer Modeling in Engineering and Sciences; 69:167-197, 2010.

[16] Buroni FC, Ortiz JE, Sáez A. Multiple pole residue approach for 3D BEM analysis of mathematical degenerate and non-degenerate materials. International Journal for Numerical Methods in Engineering; 86:1125-1143, 2011.

[17] Pan Y, Chou T. Point force solution for an infinite transversely isotropic solid. Journal of Applied Mechanics; 98:608-612, 1976.

[18] Sáez A, Ariza MP, Domínguez J. Three-dimensional fracture analysis in tranversely isotropic solids. Engineering Analysis with Boundary Elements; 20:287-298, 1997.

[19] Pan E, Yuan GF. Boundary element analysis of three-dimensional cracks in anisotropic solids. International Journal for Numerical Methods in Engineering; 48:211-237, 2000.

[20] Loloi M. Boundary integral equation solution of three-dimensional elastostatic problems in transversely isotropic solids using closed-form displacement fundamental solutions. International Journal for Numerical Methods in Engineering; 48:823$842,2000$. 
[21] Ding HJ, Liang J, Chen B. The unit point force solution for both isotropic and transversely isotropic media. Communications in Numerical Methods in Engineering; 13:95-102, 1997.

[22] Yue ZQ. Elastic fields in two joined transversely isotropic solids due to concentrated forces. International Journal of Engineering Science; 33:351-369, 1995.

[23] Ariza MP, Domínguez J. Boundary element formulation for 3D transversely isotropic cracked bodies. International Journal for Numerical Methods in Engineering; 60:719-753, 2004.

[24] París, F, Cañas, J., Boundary Element Method, Fundamentals and Applications. OUP: Oxford, 1997.

[25] Aliabadi MH. The Boundary Element Method, Volume 2 Applications in Solids and Structures. John Wiley \& Sons: Chichester, 2002.

[26] Sutradhar A, Paulino G, Gray L. Symmetric Galerkin Boundary Element Method. Springer: Berlin, 2008. 